\title{
Support and separation properties of convex sets in finite dimension
}

\author{
V. Soltan \\ Department of Mathematical Sciences, George Mason University \\ 4400 University Drive, Fairfax, VA 22030, USA
}

vsoltan@gmu.edu

Received August 21, 2021

Accepted September 20, 2021

Presented by H. Martini

Abstract: This is a survey on support and separation properties of convex sets in the $n$-dimensional Euclidean space. It contains a detailed account of existing results, given either chronologically or in related groups, and exhibits them in a uniform way, including terminology and notation. We first discuss classical Minkowski's theorems on support and separation of convex bodies, and next describe various generalizations of these results to the case of arbitrary convex sets, which concern bounding and asymptotic hyperplanes, and various types of separation by hyperplanes, slabs, and complementary convex sets.

Key words: Convex, cone, bound, hyperplane, support, separation.

MSC (2020): 52A20.

\section{Contents}

$\begin{array}{lll}1 & \text { Introduction } & 242\end{array}$

$\begin{array}{lll}2 & \text { Preliminaries } & 243\end{array}$

3 Minkowski's theorems 247

3.1 Support hyperplanes . . . . . . . . . . . . . . . . . . 247

3.2 Bounding and separating hyperplanes . . . . . . . . . . . . 250

3.3 Sufficient conditions for convexity of solid sets . . . . . . . . . 252

4 Supports and bounds of convex sets 254

4.1 Support hyperplanes . . . . . . . . . . . . . . . . . . . 254

4.2 Bounding hyperplanes and halfspaces . . . . . . . . . . . . 256

5 Separation of convex sets 260

5.1 Classification of separating hyperplanes . . . . . . . . 260

ISSN: 0213-8743 (print), 2605-5686 online

(C) The author(s) - Released under a Creative Commons Attribution License (CC BY-NC 3.0) 
5.2 Geometric conditions on hyperplane separation . . . . . . . . 264

5.3 Sharp separation of convex cones . . . . . . . . . . . . . 267

5.4 Penumbras and separation . . . . . . . . . . . . . . . 269

5.5 Hemispaces $\ldots \ldots \ldots \ldots \ldots$. . . . . . . . . . . . . . 271

\section{INTRODUCTION}

Support and separation properties of convex sets are among the core topics of convexity theory. Introduced and studied by the prominent mathematician Hermann Minkowski (see 63] and [64]), they became useful tools of convex geometry. Further development of Minkowski's ideas occurred at the beginning of 20th century and was summarized in the monograph of Bonnesen and Fenchel [8].

A rapid growth of linear analysis in the first half of 20th century led to numerous generalizations of Minkowski's contribution to the case of infinitedimensional vector spaces and made this topic an organic part of the discipline. For instance, the survey of Klee [51] from 1969 describes existing results on support and separation properties of convex sets in vector spaces of any dimension, with sporadic divisions into finite- and infinite-dimensional cases. However, besides obvious similarities between these cases, they display nowadays different goals: while finite-dimensional convexity deals, predominantly, with the properties of convex sets, a big part of similar results in linear analysis became a tool for various classifications of topological and normed spaces.

A new wave of interest towards finite-dimensional convexity occurred in the second half of the 20th century due to advancements in linear programming, with polyhedral sets considered as geometric interpretation of solutions sets of systems of linear inequalities (see, e.g., Dantzig [19] and Černikov [18]), and later in convex analysis, optimization theory, and polyhedral geometry. Various books on convex analysis (see, e.g., Güler [37, Panik [69], and Rockafellar [72]) contain separate chapters on support and separation properties of convex sets, illustrating a continuous development of these topics. These books and numerous articles in the field also underline a shift of interest from the study of convex bodies toward arbitrary convex sets, possibly unbounded or nonclosed.

Despite a steady progress in research, no comprehensive survey on support and separation properties of convex sets in finite dimension was published during the last five decades. The present paper aims to fill in (at least partly) this 
gap and overview new trends and results. It contains an account of existing facts, given either chronologically or in related groups, exhibiting them in a uniform way, including terminology and notation. We do not consider here algorithmic and computational aspects of the theory on support and separation of convex sets, which deserve their own surveys.

Following the necessary preliminaries, given in Section 2, the main text is divided into three parts. Section 3 describes classical Minkowski's theorems on support and separation properties of convex bodies and summarizes immediate contributions of his colleagues. Contemporary approach to the study of these topics is given in Sections 4 and 5. These sections cover existing results on support, bounding and asymptotic planes of arbitrary convex sets, and various types of separation of convex sets by hyperplanes, slabs, and complementary convex halfspaces.

It is interesting to compare methods of research on support and separation of convex sets in classical and contemporary periods. Since the main results of the classical period are related to full-dimensional compact sets, the methods of their proofs are predominantly based on compactness arguments and basic topology of open sets in finite dimension. Contemporary results in this field deal with arbitrary convex sets, possibly unbounded and having intermediate dimension. Consequently, their proofs employ the concept of relative interior and extensively use various types of cones associated with convex sets.

\section{Preliminaries}

This section describes necessary notation, terminology, and results on convex sets in the $n$-dimensional Euclidean space $\mathbb{R}^{n}$ (see, e.g., [76] for details). The elements of $\mathbb{R}^{n}$ are called vectors (or points), and $o$ stands for the zero vector of $\mathbb{R}^{n}$. An $r$-dimensional plane $L$ in $\mathbb{R}^{n}$, where $0 \leq r \leq n$, is a translate, $L=a+S, a \in \mathbb{R}^{n}$, of a suitable $r$-dimensional subspace $S$ of $\mathbb{R}^{n}$, called the direction space of $L$. A hyperplane is a plane of dimension $n-1$; it can be described as

$$
H=\left\{x \in \mathbb{R}^{n}: x \cdot e=\gamma\right\}, \quad e \neq o, \gamma \in \mathbb{R},
$$

where $x \cdot e$ means the dot product of vectors $x$ and $e$. Nonzero multiples $\lambda e$ of the vector $e$ in (2.1) are called normal vectors of $H$. The direction space of the hyperplane $(2.1)$ is the $(n-1)$-dimensional subspace given by

$$
S=\left\{x \in \mathbb{R}^{n}: x \cdot e=0\right\}, \quad e \neq o .
$$


Every hyperplane of the form (2.1) determines the opposite closed halfspaces

$$
V_{1}=\left\{x \in \mathbb{R}^{n}: x \cdot e \leq \gamma\right\} \quad \text { and } \quad V_{2}=\left\{x \in \mathbb{R}^{n}: x \cdot e \geq \gamma\right\}
$$

and the pair of opposite open halfspaces

$$
W_{1}=\left\{x \in \mathbb{R}^{n}: x \cdot e<\gamma\right\} \quad \text { and } \quad W_{2}=\left\{x \in \mathbb{R}^{n}: x \cdot e>\gamma\right\} .
$$

The closed and open (line) segments with distinct endpoints $u$ and $v$ in $\mathbb{R}^{n}$ and the halfline through $v$ with endpoint $u$ are denoted by $[u, v],(u, v)$, and $[u, v\rangle$, respectively. The norm (or the length) of a vector $x \in \mathbb{R}^{n}$ is denoted $\|x\|$. Given a point $a \in \mathbb{R}^{n}$ and a scalar $\rho>0$, the sphere and balls (closed and open) of radius $\rho$ and center $a$ are denoted $S_{\rho}(a), B_{\rho}(a)$, and $U_{\rho}(a)$, respectively.

The topological interior, closure, and boundary of a nonempty set $X \subset \mathbb{R}^{n}$ are given by $\operatorname{int} X, \operatorname{cl} X$, and bd $X$, respectively. The open $\rho$-neighborhood of $X$, denoted $U_{\rho}(X)$, is the union of all open balls $U_{\rho}(x)$ of radius $\rho>0$ centered at $x \in X$. Nonempty sets $X_{1}$ and $X_{2}$ in $\mathbb{R}^{n}$ are called strongly disjoint provided $U_{\rho}\left(X_{1}\right) \cap U_{\rho}\left(X_{2}\right)=\varnothing$ for a suitable $\rho>0$; the latter occurs if and only if the inf-distance $\delta\left(X_{1}, X_{2}\right)$, defined by

$$
\delta\left(X_{1}, X_{2}\right)=\inf \left\{\left\|x_{1}-x_{2}\right\|: x_{1} \in X_{1}, x_{2} \in X_{2}\right\},
$$

is positive. For a nonempty set $X \subset \mathbb{R}^{n}$, the notations span $X$ and $X^{\perp}$, stand, respectively, for the span and orthogonal complement of $X$. The set $X$ is called proper if $\varnothing \neq X \neq \mathbb{R}^{n}$.

The affine span of $X$, denoted aff $X$, is the intersection of all planes containing $X$, and $\operatorname{dim} X$ is defined as the dimension of the plane aff $X$. Also, the direction space of $X$ is defined by $\operatorname{dir} X=\operatorname{aff} X-\operatorname{aff} X$, and the orthogonal space of $X$ by $\operatorname{ort} X=(\operatorname{dir} X)^{\perp}$ (generally, ort $\left.X \neq X^{\perp}\right)$. Given nonempty sets $X, Y \subset \mathbb{R}^{n}$ and a scalar $\lambda$, we let

$$
X+Y=\{x+y: x \in X, y \in Y\}, \quad \lambda X=\{\lambda x: x \in X\} .
$$

Nonempty sets $X$ and $Y$ in $\mathbb{R}^{n}$ are called (directly) homothetic provided $X=z+\lambda Y$ for suitable $z \in \mathbb{R}^{n}$ and $\lambda>0$.

In what follows, $K$ means a convex set in $\mathbb{R}^{n}$. To avoid trivial cases, we will be assuming that all convex sets involved are nonempty. A point $x$ of a convex set $K \subset \mathbb{R}^{n}$ is said to be relatively interior to $K$ provided there is a scalar $\rho=\rho(x)>0$ such that aff $K \cap U_{\rho}(x) \subset K$. The set of all relatively 
interior points of $K$ is called the relative interior of $K$ and is denoted rint $K$. The set $\operatorname{rint} K$ is nonempty and convex; furthermore, $\operatorname{cl} K=\operatorname{cl}(\operatorname{rint} K)$. The difference between topological and relative interiors can be illustrated by the following example: If $K$ is a unit circular disk of the coordinate $x y$-plane of $\mathbb{R}^{3}$, then int $K=\varnothing$, while rint $K$ is the interior of this disk. The relative boundary of a convex set $K \subset \mathbb{R}^{n}$, denoted $\operatorname{rbd} K$, is defined by $\operatorname{rbd} K=\operatorname{cl} K \backslash \operatorname{rint} K$. It is known that $\operatorname{rbd} K \neq \varnothing$ if and only if $K$ is not a plane.

A convex body in $\mathbb{R}^{n}$ is a compact convex set with nonempty interior, a (convex) polyhedron is the intersection of finitely many closed halfspaces, and a polytope (bounded polyhedron) is the convex hull of finitely many points.

A contemporary approach to the study of support and separation properties of convex sets deals with a family of various associated cones. We recall that a nonempty set $C$ in $\mathbb{R}^{n}$ is called a cone with apex $a \in \mathbb{R}^{n}$ if $a+\lambda(x-a) \in C$ whenever $\lambda \geq 0$ and $x \in C$. (Obviously, this definition implies that $a \in C$, although a stronger condition $\lambda>0$ can be beneficial; see, e.g., [57].) The cone $C$ is called convex if it is a convex set. The apex set of a convex cone $C \subset \mathbb{R}^{n}$, denoted ap $C$, is the set of all apices of $C$. If $a$ is an apex of a convex cone $C$, then ap $C=C \cap(2 a-C)$. Natural generalizations of cones are given by the sets of the form $K=B+C$, where $B$ is a compact convex set and $C$ is a convex cone; these sets are called M-decomposable if $C$ is closed, and M-predecomposable otherwise (see Goberna et al. 35] and Iusemi et al. [41, respectively).

For a convex set $K \subset \mathbb{R}^{n}$ and a point $a \in \mathbb{R}^{n}$, the generated cone $C_{a}(K)$ with apex $a$ is defined by

$$
C_{a}(K)=\{a+\lambda(x-a): x \in K, \lambda \geq 0\} .
$$

Both sets $C_{a}(K)$ and $\operatorname{cl} C_{a}(K)$ are convex cones with apex $a$ (we observe that the cone $C_{a}(K)$ may be nonclosed even if $K$ is closed). Furthermore, $C_{a}(K)$ is a plane if and only if $a \in \operatorname{rint} K$. In particular, $C_{a}(K)=\mathbb{R}^{n}$ if and only if $a \in \operatorname{int} K$.

The recession cone of a convex set $K \subset \mathbb{R}^{n}$ is defined by

$$
\operatorname{rec} K=\left\{e \in \mathbb{R}^{n}: x+\lambda e \in K \text { whenever } x \in K \text { and } \lambda \geq 0\right\} .
$$

If $K$ is closed, then rec $K$ is a closed convex cone with apex $o$, and $\operatorname{rec} K \neq\{o\}$ if and only if $K$ is unbounded. The lineality space of $K$ is the subspace defined by $\operatorname{lin} K=\operatorname{rec} K \cap(-\operatorname{rec} K)$.

In a standard way, the (negative) polar cone of a convex set $K \subset \mathbb{R}^{n}$ is defined by

$$
K^{\circ}=\left\{e \in \mathbb{R}^{n}: x \cdot e \leq 0 \text { for all } x \in K\right\} .
$$


The set $K^{\circ}$ is a closed convex cone with apex $o$. Furthermore,

1. $K^{\circ}=\{o\}$ if and only if $o \in \operatorname{int} K$, and $K^{\circ}$ is a subspace if and only if $o \in \operatorname{rint} K$.

2. $K^{\circ}=\left(C_{o}(K)\right)^{\circ},\left(K^{\circ}\right)^{\circ}=\operatorname{cl} C_{o}(K), \operatorname{lin} K^{\circ}=K^{\perp}$.

3. If $C \subset \mathbb{R}^{n}$ is a closed convex cone with apex $o$, then a nonzero vector $e \in \mathbb{R}^{n}$ belongs to rint $C^{\circ}$ if and only if $x \cdot e<0$ for all $x \in C \backslash \operatorname{lin} C$.

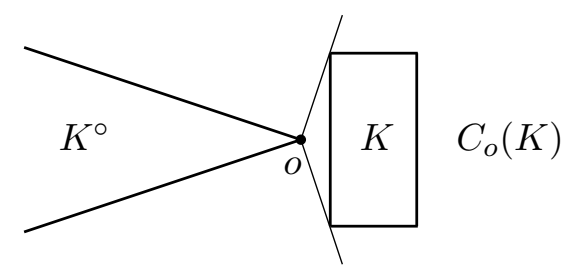

Figure 1: The polar cone of a convex set $K$.

Given a point $z$ in the closure of a convex set $K \subset \mathbb{R}^{n}$, the polar cone $(K-z)^{\circ}$ is often called the normal cone of $K$ at $z$ and is denoted $N_{z}(K)$; it consists of all vectors $e \in \mathbb{R}^{n}$ such that $z$ is the nearest to $e+z$ point in $\operatorname{cl} K$.

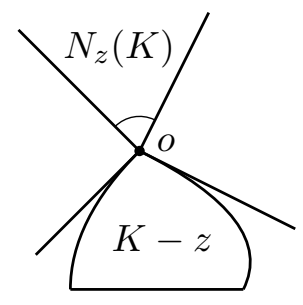

Figure 2: The normal cone $N_{z}(K)$.

The union of all normal cones $N_{z}(K)$, where $z \in \operatorname{cl} K$, is denoted nor $K$ and called the normal cone of $K$. The set nor $K$ is a cone with apex $o$, which is not necessarily closed or convex. One more cone associated with $K$ is its barrier cone, defined as

bar $K=\left\{e \in \mathbb{R}^{n}: \exists \gamma=\gamma(e) \in \mathbb{R}\right.$ such that $x \cdot e \leq \gamma$ for all $\left.x \in K\right\}$. 
It is known that bar $K$ is a convex (not necessarily closed) cone with apex $o$. Generally,

$$
\operatorname{rint}(\operatorname{rec}(\operatorname{cl} K))^{\circ} \subset \operatorname{nor} K \subset \operatorname{bar} K \subset(\operatorname{rec}(\operatorname{cl} K))^{\circ},
$$

which implies the equalities $\operatorname{rec}(\operatorname{cl} K)=(\text { nor } K)^{\circ}=(\text { bar } K)^{\circ}$. For instance, if $K=\left\{(x, y): y \geq x^{2}\right\}$, then $K^{\circ}=\{(0, y): y \leq 0\}$ and

$$
\begin{aligned}
& \operatorname{rec} K=\{(0, y): y \geq 0\}, \quad(\operatorname{rec} K)^{\circ}=\{(x, y): y \leq 0\}, \\
& \operatorname{nor} K=\operatorname{bar} K=\{o\} \cup\{(x, y): y<0\} .
\end{aligned}
$$

A point $z$ of a convex set $K \subset \mathbb{R}^{n}$ is called an extreme point of $K$ provided the set $K \backslash\{z\}$ is convex (equivalently, the equality $z=(1-\lambda) u+\lambda v$, where $u, v \in K$ and $0<\lambda<1$, is possible only if $u=v=z$ ). Similarly, $z$ is an exposed point of $K$ provided there is a hyperplane $H \subset \mathbb{R}^{n}$ satisfying the condition $H \cap K=\{z\}$. The sets ext $K$ and $\exp K$ of extreme and exposed points of a closed convex set $K \subset \mathbb{R}^{n}$ have the following properties.

4. $\exp K \neq \varnothing \Leftrightarrow \operatorname{ext} K \neq \varnothing \Leftrightarrow K$ is line-free (that is, $K$ contains no line).

5. $\exp K \subset \operatorname{ext} K \subset \operatorname{cl}(\exp K)$.

\section{Minkowski's Theorems}

3.1. Support Hyperplanes The concept of support hyperplane is attributed to Minkowski [63, §8]: Given a nonempty set $X \subset \mathbb{R}^{n}$, a hyperplane $H \subset \mathbb{R}^{n}$ supports $X$ if it contains at least one point of $X$ and does not cut $X$ (that is no two points of $X$ belong, respectively, to the opposite open halfspaces determined by $H$ ). Clearly, the above condition " $H$ does not cut $X$ " can be equivalently reformulated as " $X$ entirely lies in a closed halfspace determined by $H . "$ The following well-known result is due to Minkowski (see [63, §16] for all $n \geq 3$ and [64, pp. $139-141]$ for $n=3$ ).

Theorem 3.1. ([63, § 16]) Every boundary point of a convex body $K \subset$ $\mathbb{R}^{n}$ belongs to a hyperplane supporting $K$.

Since general theory of convex sets, with credible geometric arguments, was still in rudimentary stage, Minkowski's proof of Theorem 3.1 used an analytic description of convex bodies in terms of radial distances (see 63, $\S 1]$ ). The radial distance (which later became known as the Minkowski gauge function) from a point $a$ to a point $b$ in $\mathbb{R}^{n}$ is a real-valued function $S(a, b)$ satisfying the following conditions: 
1. $S(a, b)>0$ if $a \neq b$, and $S(a, a)=0$;

2. if $c=a+t(b-a)$, where $t \geq 0$, then $S(a, c)=t S(a, b)$;

3. $S(a, c) \leq S(a, b)+S(b, c)$ whenever $a, b, c \in \mathbb{R}^{n}$.

The standard surface $F$ and the standard body $K$ of the radial distance function $S(o, x)$ are defined, respectively, by

$$
F=\left\{x \in \mathbb{R}^{n}: S(o, x)=1\right\} \quad \text { and } \quad K=\left\{x \in \mathbb{R}^{n}: S(o, x) \leq 1\right\} .
$$

Minkowski observed (see [63, §8]) that the standard body $K$ from (3.1) is a compact convex set containing the origin $o$ in its interior, and, conversely, that a certain translate of a convex body can be viewed as the standard body of a suitable radial distance function. Using the properties of $S(a, b)$, and not the convexity of $K$, Minkowski showed that any point of $F$ belongs to a hyperplane supporting $F$ (and thus supporting $K$ ). His proof consists of two steps.

Step 1. Given a point $z \in F$ and a scalar $t>1$, there is a hyperplane $H(t)$ contained in $\mathbb{R}^{n} \backslash K$ and meeting the open interval $(z, t z)$ (see Figure 3 ).

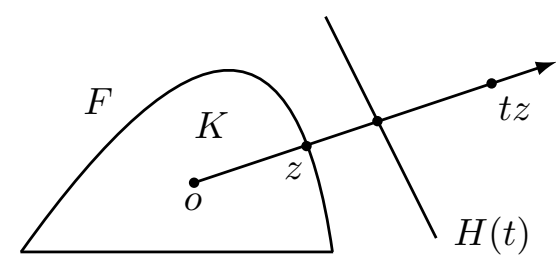

Figure 3: A hyperplane $H(t)$ in $\mathbb{R}^{n} \backslash K$.

Step 2. Given a sequence of scalars $t_{1}, t_{2}, \ldots(>1)$ tending to 1 and a respective sequence of hyperplanes $H\left(t_{1}\right), H\left(t_{2}\right), \ldots$ obtained in the above Step 1 and described as

$$
\beta_{0}\left(t_{i}\right)+\beta_{1}\left(t_{i}\right) x_{1}+\cdots+\beta_{n}\left(t_{i}\right) x_{n}=0, \quad i \geq 1,
$$

one can choose $n+1$ infinite subsequences $\beta_{j}\left(t_{i_{1}}\right), \beta_{j}\left(t_{i_{2}}\right), \ldots, 0 \leq j \leq n$, which converge, respectively, to scalars $\beta_{0}, \beta_{1}, \ldots, \beta_{n}$ such that the limit hyperplane $\beta_{0}+\beta_{1} x_{1}+\cdots+\beta_{n} x_{n}=0$ supports $F$ at $z$.

The analytic nature of Minkowski's proof prompted various mathematicians to consider more geometric approaches. For instance, Carathéodory [16] proved Theorem 3.1 based on the following auxiliary result, afterward widely used in convex geometry (see Figure 4). 
Theorem 3.2. ([16]) Let $K \subset \mathbb{R}^{n}$ be a convex body, and $u$ be a point outside $K$. If $z$ is the nearest to $u$ point in $K$, then the hyperplane $H$ through $z$ orthogonal to the segment $[u, z]$ supports $K$ such that $u$ and $K$ lie in the opposite closed halfspaces determined by $H$.

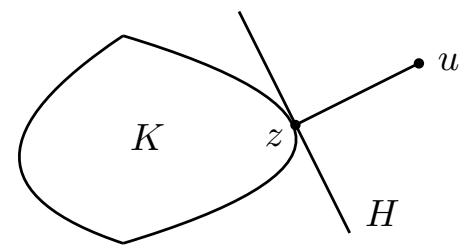

Figure 4: Illustration to Theorem 3.2 .

Straszewicz [86, pp. 19-21] gave one more method of the proof of Theorem 3.1 which uses an induction argument. Steinitz (see [83, §11] and [84, §26]) showed that Theorem 3.1 and Theorem 3.2 hold for the case of any proper $n$-dimensional convex set $K \subset \mathbb{R}^{n}$, but his contribution went unnoticed.

Various proofs of Theorem 3.1 for the case of dimensions 2 and 3 were provided at that time. For instance, Brunn [10] uses a similar to Straszewicz [86] method (and later another method in [11]), while Blaschke [7, pp. 53-54] finds a suitable orthogonal projection of a convex body $K \subset \mathbb{R}^{3}$ on a plane and uses the support property of this projection in the plane.

We observe that in all three sources [16, 63, 86], the resulting support hyperplane of a given convex body is obtained as the limit of a suitable converging sequence of hyperplanes. Proofs of Theorem 3.1 which do not employ limit procedures appeared much later. These can be found in the papers of Favard [28, Chapter 2] and Botts [9], as given below.

THEOREM 3.3. ([9]) If $z$ is a boundary point of a compact convex set $K \subset \mathbb{R}^{n}$, and $v$ is a point of the unit sphere $S_{1}(z)$ at a largest distance from $K$, then the hyperplane through $z$ orthogonal to the segment $[v, z]$ supports $K$.

Support properties of convex bodies are used for various classifications of their boundary points. For instance, a point $z$ of a convex body $K \subset \mathbb{R}^{n}$ is called regular if it belongs to a unique support hyperplane of $K$. It is wellknown that the set of regular points of a convex body $K \subset \mathbb{R}^{n}$ is everywhere 
dense in bd $K$. Historical references here are due to Jensen 44 and Bernstein [4, 5], (for the planar case), Kakeya [45, Fujiwara [31, and Reidemeister [71] (for the 3-dimensional case), and Mazur [62] (for the case of linear normed spaces of any dimension).

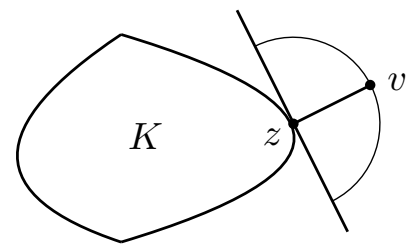

Figure 5: Illustration to Theorem 3.3 .

Botts [9] gave the following characteristic property of regular points: If $K \subset \mathbb{R}^{n}$ is a convex body, $z_{0} \in \mathrm{bd} K$, and $H$ is a hyperplane supporting $K$ at $z_{0}$, then $z_{0}$ is regular if and only if for every sequence of points $z_{1}, z_{2}, \ldots \in \operatorname{bd} K \backslash\left\{z_{0}\right\}$ converging to $z_{0}$, the sequence of numbers

$$
\frac{\delta\left(z_{1}, H\right)}{\left\|z_{1}-z_{0}\right\|}, \frac{\delta\left(z_{2}, H\right)}{\left\|z_{2}-z_{0}\right\|}, \ldots
$$

tends to 0 , where $\delta\left(z_{i}, H\right)$ denotes the distance from $z_{i}$ to $H$.

One more classification of boundary points derives from observing contact sets of a convex body $K \subset \mathbb{R}^{n}$ and its support hyperplanes. For example, the equality $K=\operatorname{cl}(\operatorname{conv}(\exp K))$, proved by Straszewicz [87, implies that $K$ has at least $n+1$ exposed points, and that the set $\exp K$ is finite if and only if $K$ is a polytope.

3.2. Bounding and Separating Hyperplanes An important class of hyperplanes with respect to a given convex body was considered by Carathéodory [17] and used by him to describe the closed convex hull of a compact set in $\mathbb{R}^{n}$. Namely, a hyperplane $H \subset \mathbb{R}^{n}$ is said to bound a convex body $K \subset \mathbb{R}^{n}$ provided $H \cap K=\varnothing$. Equivalently, $H$ bounds $K$ if $K$ lies in one of the open halfspaces determined by $H$ (see Figure 6).

Theorem 3.4. ([17]) If $z \in \mathbb{R}^{n}$ is an exterior point of a convex body $K \subset \mathbb{R}^{n}$, and $u$ is the nearest to $z$ point in $K$, then the hyperplane through $z$ orthogonal to the segment $[u, z]$ bounds $K$. 
The following separation theorem of Minkowski [64] originated a variety of related results.

Theorem 3.5. ([64, p. 141]) If $K_{1}$ and $K_{2}$ are convex bodies in $\mathbb{R}^{3}$ with disjoint interiors, then there is a plane $H$ such that the sets $\operatorname{int} K_{1}$ and $\operatorname{int} K_{2}$ belong to the opposite open halfspaces determined by $H$.

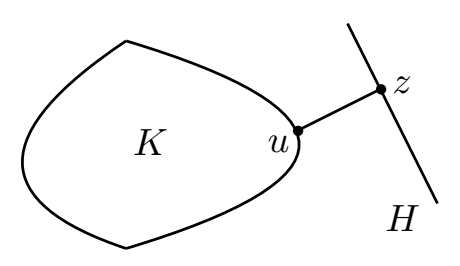

Figure 6: Bounding hyperplane of $K$ through a given point $z$.

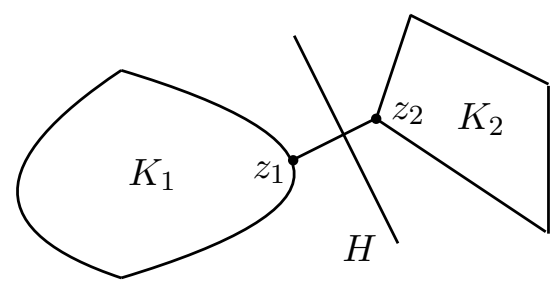

Figure 7: Separating hyperplane of convex bodies $K_{1}$ and $K_{2}$.

Minkowski's proof of this theorem is divided into two cases.

Case 1: $K_{1} \cap K_{2}=\varnothing$. If points $z_{1} \in K_{1}$ and $z_{2} \in K_{2}$ are at a minimum possible distance, and if $H$ is a plane orthogonal to the segment $\left[z_{1}, z_{2}\right]$ and passing through an interior point of this segment, then $K_{1}$ and $K_{2}$ are contained in the opposite open halfspaces determined by $H$ (see Figure 7 ).

Case 2: $K_{1} \cap K_{2} \neq \varnothing$. Assuming that $o \in \operatorname{int} K_{1}$, any smaller homothetic copy $t K_{1}$ of $K_{1}$, where $0<t<1$, is disjoint from $K_{2}$. By the above Case 1 , there is a plane $H(t)$ such that $t K_{1}$ and $K_{2}$ belong to the opposite open halfspaces determined by $H(t)$. Given a sequence of scalars $t_{1}, t_{2}, \ldots$ in the interval $(0,1)$ which tend to 1 , one can chose a sequence of planes $H\left(t_{i}\right)$ separating, respectively, $t_{i} K_{1}$ and $K_{2}$ and converging to a plane $H$ such that $K_{1}$ and $K_{2}$ belong to the opposite closed halfspaces determined by $H$. 
Interestingly, Minkowski did not use the term "separating plane". This term ("Zwischenebene") was used later by Brunn [12. The assertion of Theorem 3.5 was formulated by Bonnesen and Fenchel [8, p. 5] for the $n$-dimensional case, with the term "separating hyperplane", without any reference on Minkowski [64. Theorem 3.5 was generalized in 1936 by Eidelheit [25] for the case of convex bodies in linear normed spaces of any dimension (see the survey [51] for further bibliography).
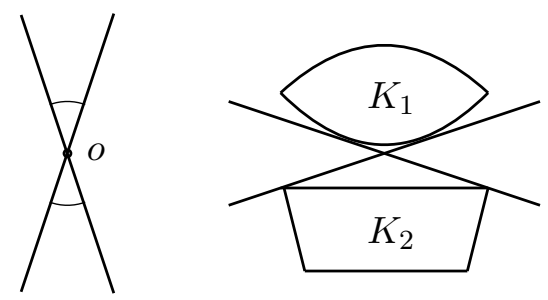

Figure 8: Double cone of normal vectors to separating hyperplanes.

The following result, obtained by Brunn [12], describes the normal vectors of all planes which separate a given pair of convex bodies (compare with Theorem 5.3 below).

Theorem 3.6. ([12]) Let $K_{1}$ and $K_{2}$ be disjoint convex bodies in $\mathbb{R}^{3}$. The set of normal vectors (drawn at the origin of $\mathbb{R}^{3}$ ) to all planes separating $K_{1}$ and $K_{2}$ is a convex double cone, i.e., is the union of two opposite convex cones with the improper common apex o (see Figure 8).

3.3. Sufficient Conditions for Convexity of Solid Sets The following theorem of Minkowski 63] characterizes bounded convex surfaces in terms of their support property.

Theorem 3.7. ([63, §17]) A set $F \subset \mathbb{R}^{n}$ is the boundary of a convex body in $\mathbb{R}^{n}$ provided there is a point $z \in \mathbb{R}^{n}$ such that

(a) every open halfline originated at $z$ meets $F$,

(b) every point of $F$ belongs to a hyperplane supporting $F$.

Using similar arguments, Brunn [10, p. 293] proved the theorem below. 
Theorem 3.8. ([10]) If a compact set $X \subset \mathbb{R}^{n}$ has nonempty interior and any boundary point of $X$ belongs to a hyperplane supporting $X$, then $X$ is a convex body.

Straszewicz [86, pp. 22-24] independently proved Theorem 3.8 under the additional assumption that $X$ is connected and coincides with the closure of its interior. A similar result was obtained by Haalmeijer [38]: If $X \subset \mathbb{R}^{n}$ is the closure of an open connected set and $Y$ is a dense subset of bd $X$, then $X$ is convex provided every point $x \in Y$ belongs to a hyperplane supporting $X$.

Tietze (see [89] and [90]) gave two local versions of Theorem 3.8. Following [89], an open half-ball $Q_{\rho}(z) \subset \mathbb{R}^{n}$ with center $z$ and radius $\rho>0$ means the intersection of the open ball $U_{\rho}(z)$ and an open halfspace whose boundary hyperplane contains $z$.

Theorem 3.9. ([89, 90]) A compact set $X \subset \mathbb{R}^{n}$ is convex if it satisfies any of the following two conditions:

(a) $X$ is the closure of an open connected set, and for every boundary point $z$ of $X$ there is an open half-ball $Q_{\rho}(z), \rho=\rho(z)>0$, disjoint from $X$;

(b) $X$ is connected, int $X \neq \varnothing$, and there is a scalar $\rho>0$ such that for every boundary point $z$ of $X$ a suitable open half-ball $Q_{\rho}(z)$ is disjoint from $X$.

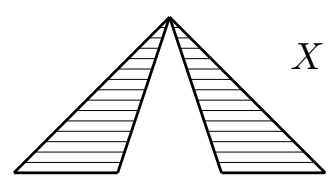

Figure 9: Illustration to Theorem 3.9.

The nonconvex sets $X \subset \mathbb{R}^{2}$ in Figure 9 illustrate that the assumptions in both conditions (a) and (b) of Theorem 3.9 are essential. Indeed, at any boundary point $z$, the set $X$ has a support open half-ball $Q_{\rho}(z)$, where $\rho=\rho(z)$, while int $X$ is not connected and no constant scalar $\rho>0$ satisfies condition (b).

A simplified proof of part (b) of Theorem 3.9 was given by Reinhardt [70]. Also, this part was sharpened later in the following ways. 
1. Gericke [34] (see also Nöbeling [67]) showed that the centers of disjoint with $X$ half-balls $Q_{\rho}(z)$ can be chosen in bd $X \backslash L$, where $L$ is a suitable $(n-3)$-dimensional plane.

2. Süß 88 proved that condition (b) is satisfied if $X$ is connected, has interior points, and there is a scalar $\rho>0$ such that for every boundary point $z$ of $X$ there is a cylinder $C(z)$ of variable height based on an $(n-1)$-dimensional ball of radius $\rho$, with $X \cap \operatorname{int} C(z)=\varnothing$.

3. Schmidt (see Bieberbach [6, p. 20]) observed that it is sufficient to require the existence of a hyperplane $H$ through $z$ which does not meet the set $X \cap U_{\rho}(z)$ (without the assumption that $H$ supports $X \cap U_{\rho}(z)$ ).

Burago and Zalgaller [13] (see pp. 395 and 415) observed that the word "compact" can be replaced by "closed" in both Theorem 3.8 and Theorem 3.9. Also, they changed the language of Theorem 3.9, replacing in (b) the requirement $X \cap Q_{\rho}(z)=\varnothing$ with the following one: $X \cap U_{\rho}(z)$ is supported at $z$ by a suitable hyperplane.

One more variation of Theorem 3.8 comes from geometric measure theory. Namely, a closed set $X \subset \mathbb{R}^{n}$ with non-empty interior is convex if and only if it has locally finite perimeter and possesses a support hyperplane at each point of its reduced boundary (see Caraballo [14, 15] for definitions and technical details).

\section{Supports And Bounds of Convex Sets}

4.1. Support Hyperplanes An extension of Minkowski's definition of support hyperplane says that a hyperplane $H \subset \mathbb{R}^{n}$ supports a nonempty set $X \subset \mathbb{R}^{n}$ provided $H$ meets its closure, $\operatorname{cl} X$, and does not cut $X$. Analysis of the proof of Theorem 3.3 shows that its assertion holds for the case of any proper convex set $K \subset \mathbb{R}^{n}$. Consequently, Theorem 3.1 can be generalized as follows:

TheOrem 4.1. Any boundary point of a convex set $K \subset \mathbb{R}^{n}$ belongs to a hyperplane supporting $K$.

If the dimension of a convex set $K \subset \mathbb{R}^{n}$ is less than $n$, then a hyperplane $H \subset \mathbb{R}^{n}$ supporting $K$ may contain $K$ entirely. Nevertheless, in many instances it is important to know whether $H$ properly supports $K$, that is whether $K \not \subset H$. Equivalent terms used in the literature are essential support (see Steinitz [84, §28]) and nontrivial support (see Rockafellar [72, p. 100]). 
Theorem 4.2. ([72, Theorem 11.6]) Let $K \subset \mathbb{R}^{n}$ be a convex set which is not a plane, and let $F$ be a nonempty convex subset of $\mathrm{cl} K$ (for instance, $F$ is a singleton). There is a hyperplane containing $F$ and properly supporting $K$ if and only if $F \subset \operatorname{rbd} K$.

It is easy to see that a hyperplane $H$ properly supports a convex set $K$ if and only if it meets $\mathrm{cl} K$ such that $H \cap \operatorname{rint} K=\varnothing$. The following assertion is a variation of Theorem 4.2 .

ThEOREM 4.3. ([76, Corollary 9.11]) If a plane $L \subset \mathbb{R}^{n}$ meets the closure of a convex set $K \subset \mathbb{R}^{n}$ such that $L \cap \operatorname{rint} K=\varnothing$, then there is a hyperplane containing $L$ and properly supporting $K$.

The next result shows that the existence of a support hyperplane is a local property.

Theorem 4.4. ([76, Problem 9.3]) Let $K \subset \mathbb{R}^{n}$ be a convex set, $z$ be a point in $\mathrm{cl} K$, and $B_{\rho}(z) \subset \mathbb{R}^{n}$ be a closed ball of radius $\rho>0$ centered at $z$. $A$ hyperplane $H \subset \mathbb{R}^{n}$ through $z$ supports (properly supports) $K$ if and only if $H$ supports (respectively, properly supports) the set $K \cap B_{\rho}(z)$.

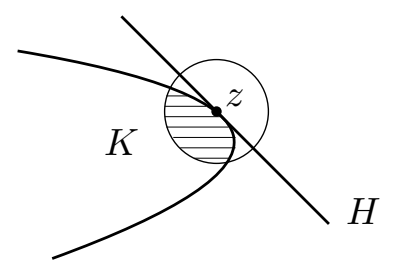

Figure 10: Local support of $K$ at $z$.

If a support hyperplane $H \subset \mathbb{R}^{n}$ of a convex set $K \subset \mathbb{R}^{n}$ is described by the equation (2.1), then its normal vector $e$ and the level scalar $\gamma$ can be characterized as follows.

1. $H$ supports (properly supports) $K$ at a point $z \in \operatorname{cl} K$ if and only if $e \in N_{z}(K)$ (respectively, $e \in N_{z}(K) \backslash$ ort $K$ ) and $\gamma=z \cdot e$.

2. $H$ supports (properly supports) $K$ if and only if $e \in$ nor $K$ (respectively, $e \in \operatorname{nor} K \backslash \operatorname{ort} K)$ and $\gamma=\sup \{x \cdot e: x \in K\}$. 
Theorem 4.5. ([76, Theorem 12.22]) If $K \subset \mathbb{R}^{n}$ is a line-free convex set, then the set of nonzero vectors $e \in \mathbb{R}^{n}$ for which the hyperplane of the form

$$
H=\left\{x \in \mathbb{R}^{n}: x \cdot e=\gamma\right\}, \quad \text { where } \gamma=\sup \{u \cdot e: u \in K\},
$$

supports $\mathrm{cl} K$ at a single point is dense in nor $K$.

One more assertion, independently proved by Durier 24] for the case of convex bodies and by Klee 52 for the case of line-free closed convex sets, complements Theorem 4.5 and the inclusion $\operatorname{ext} K \subset \operatorname{cl}(\exp K)$.

Theorem 4.6. ([24, 52]) Let $K \subset \mathbb{R}^{n}$ be a line-free closed convex set, $H$ be a hyperplane supporting $K$, and $z$ be an extreme point of $K$ that belongs to $H$. Then there is a sequence of points $z_{i} \in \exp K$ and a respective sequence of hyperplanes $H_{i}, i \geq 1$, satisfying the conditions:
(a) $H_{i} \cap K=\left\{z_{i}\right\}, i \geq 1$,
(b) $z=\lim _{i \rightarrow \infty} z_{i}$ and $H=\lim _{i \rightarrow \infty} H_{i}$.

There are a few results on support properties of special convex sets. For instance:

3. If a hyperplane $H \subset \mathbb{R}^{n}$ supports a convex cone $C \subset \mathbb{R}^{n}$, then every apex of $C$ belongs to $H$. Furthermore, the intersection of all hyperplanes supporting $C$ is precisely the apex set of $\operatorname{cl} C$ (see, e.g., [76], Theorem 9.43 and Theorem 9.46).

4. If a hyperplane $H \subset \mathbb{R}^{n}$ supports an M-predecomposable set $K \subset \mathbb{R}^{n}$, expressed as the sum of a compact convex set $B$ and a convex cone $C$ with apex $o$, then $H$ supports $B$ (see [79]).

4.2. Bounding Hyperplanes and Halfspaces Modifying Carathéodory's definition of bounding hyperplane, we say that a hyperplane $H \subset \mathbb{R}^{n}$ bounds a convex set $K \subset \mathbb{R}^{n}$ provided $K$ is contained in a closed halfspace determined by $H$. Furthermore: $H$ nontrivially bounds $K$ if $K \not \subset H$; strictly bounds $K$ if $H \cap K=\varnothing$; and strongly bounds $K$ if $H$ bounds a suitable open $\rho$-neighborhood $U_{\rho}(K)$ of $K$.

Analysis of the proof of Theorem 3.4 shows that it can be generalized in the following way. 
Theorem 4.7. Let $K$ be a proper convex set $\mathbb{R}^{n}$ and $z \in \mathbb{R}^{n} \backslash \operatorname{cl} K$. If $u$ is the nearest to $z$ point in $\mathrm{cl} K$, then the hyperplane $H \subset \mathbb{R}^{n}$ through $z$ orthogonal to the segment $[u, z]$ strongly bounds $K$ and $\delta(H, K)=\delta(z, K)=$ $\|z-u\|$.

The results below describe all hyperplanes bounding a convex set $K \subset \mathbb{R}^{n}$ and having a given direction of normal vectors or containing a given point (or even a plane) of $\mathbb{R}^{n}$. The following auxiliary statements on a hyperplane $H \subset \mathbb{R}^{n}$ immediately follow from definitions.

1. $H$ bounds $K$ if and only if $H$ can be expressed in the form (2.1), where $e \in \operatorname{bar} K$ and $\sup \{x \cdot e: x \in K\} \leq \gamma$.

2. $H$ properly bounds $K$ if and only if $H$ can be expressed in the form (2.1), where $e \in$ bar $K$ and $\gamma$ satisfies both inequalities

$$
\inf \{x \cdot e: x \in K\}<\gamma \quad \text { and } \quad \sup \{x \cdot e: x \in K\} \leq \gamma .
$$

3. $H$ strictly bounds $K$ if and only if $H$ can be expressed in the form (2.1), where $e \in \operatorname{bar} K$ and $x \cdot e<\gamma$ for all $x \in K$.

4. $H$ strongly bounds $K$ if and only if $H$ can be expressed in the form (2.1), where $e \in \operatorname{bar} K$ and $\sup \{x \cdot e: x \in K\}<\gamma$.

Theorem 4.8. ([82]) For a convex set $K \subset \mathbb{R}^{n}$ and a point $z \in \mathbb{R}^{n}$, the assertions below hold.

(a) There is a hyperplane through $z$ bounding $K$ if and only if $o \notin \operatorname{int}(K-z)$, or, equivalently, $(K-z)^{\circ} \neq\{o\}$.

(b) A hyperplane through $z$ bounds $K$ is and only if it can be expressed as

$$
H=\left\{x \in \mathbb{R}^{n}: x \cdot e=z \cdot e\right\},
$$

where $e \in(K-z)^{\circ} \backslash\{o\}$.

Theorem 4.9. ([82]) For a convex set $K \subset \mathbb{R}^{n}$ and a point $z \in \mathbb{R}^{n}$, the assertions below hold.

(a) There is a hyperplane through $z$ properly bounding $K$ if and only if $o \notin \operatorname{rint}(K-z)$, or, equivalently, $(K-z)^{\circ} \backslash \operatorname{lin}(K-z)^{\circ} \neq \varnothing$.

(b) A hyperplane through $z$ properly bounds $K$ if and only if it can be expressed in the form (4.1), where $e \in(K-z)^{\circ} \backslash \operatorname{lin}(K-z)^{\circ}$. 
Theorem 4.10. ([82]) For a closed convex set $K \subset \mathbb{R}^{n}$ and a point $z \in \mathbb{R}^{n}$, the assertions below hold.

(a) There is a hyperplane through $z$ strongly bounding $K$ if and only if $o \notin \operatorname{cl}(K-z)$.

(b) If $o \notin \mathrm{cl}(K-z)$ and $e \in \operatorname{rint}(K-z)^{\circ}$, then the hyperplane (4.1) strongly bounds $K$.

(c) If a hyperplane through $z$ strongly bounds $K$, then it can be expressed in the form (4.1), where $e \in(K-z)^{\circ} \backslash \operatorname{lin}(K-z)^{\circ}$. If, additionally, $K$ is compact, then $e$ can be chosen in $\operatorname{rint}(K-z)^{\circ}$.

There are various extensions of Theorem 3.4 to the case of hyperplanes bounding a convex set $K \subset \mathbb{R}^{n}$ and containing a given plane $L \subset \mathbb{R}^{n}$.

5. If $L$ does not meet the relative interior of $K$, then there is a hyperplane through $L$ properly bounding $K$ (Rockafellar [72, Theorem 11.2]).

6. If the boundary of $K$ does not contain a halfline and $L$ is disjoint from $\mathrm{cl} K$, then there is a hyperplane through $L$ strictly bounding $\mathrm{cl} K$ (Klee [47]).

7. If $\delta(K, L)>0$, then there is a hyperplane $H$ through $L$ strongly bounding $K$ such that $\delta(K, H)=\delta(K, L)$ ([76, Theorem 9.6]).

The families of hyperplanes through a plane $L \subset \mathbb{R}^{n}$ bounding (properly, strictly, or strongly) a given convex set $K \subset \mathbb{R}^{n}$ can be described similarly to Theorems 4.8 4.10. For instance,

8. There is a hyperplane which bounds $K$ and contains $L$ if and only if $o \notin \operatorname{int}(K-L)$, or, equivalently, $(K-L)^{\circ} \neq\{o\}$.

9. A hyperplane $H \subset \mathbb{R}^{n}$ bounds $K$ and contains $L$ if and only if $H$ can be expressed in the form (4.1), where $e \in(K-L)^{\circ} \backslash\{o\}$ and $z$ is any point in $L$.

We recall (see, e.g., Gale and Klee [33]) that a plane $L \subset \mathbb{R}^{n}$ is an asymptote of a set $X \subset \mathbb{R}^{n}$ provided $\operatorname{cl} X \cap L=\varnothing$ and $\delta(X, L)=0$. Existence of plane asymptotes is closely related to the properties of various algebraic operations on sets (see, e.g., Auslender and Teboulle [1] and [75], 76] for further references). For instance, the following assertions hold.

10. For a closed set $X \subset \mathbb{R}^{n}$ and a plane $L \subset \mathbb{R}^{n}$, the sum $X+L$ is closed if and only if there is a translate of $L$ which is an asymptote of $X$. 


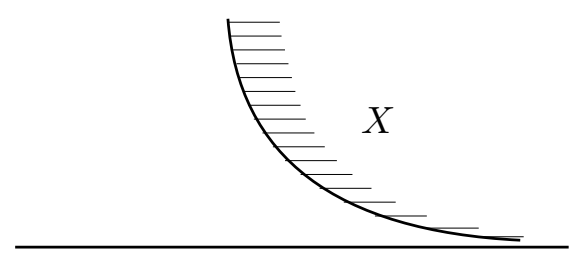

Figure 11: A plane asymptote of a set $X$.

11. For a linear transformation $f: \mathbb{R}^{n} \rightarrow \mathbb{R}^{m}$ and a closed set $X \subset \mathbb{R}^{n}$, the set $f(X)$ is closed if and only if there is a translate of null $f$ which is an asymptote of $X$.

The family of plane asymptotes of a given convex set is not hereditary. For example, if $K$ is the convex set in $\mathbb{R}^{3}$ be given by

$$
K=\left\{(x, y, z): x \geq 0, x y \geq 1, z \geq(x+y)^{2}\right\},
$$

then the $x y$ - and $x z$-coordinate planes are the only plane asymptotes of $K$. Asymptotic properties of convex sets without boundary halflines are studied by Klee [49], and of cones and M-predecomposable sets in [77] and [79]. As proved in [81], every plane asymptote $L$ of a convex set $K \subset \mathbb{R}^{n}$ contains a line-free closed convex cone which is an asymptote of $K$.

The following assertions immediately follow the above definitions.

12. Any hyperplane $H \subset \mathbb{R}^{n}$ disjoint from a closed set $X \subset \mathbb{R}^{n}$ either strongly bounds $X$ or is an asymptote of $X$.

13. A hyperplane $H \subset \mathbb{R}^{n}$ is an asymptote of a closed convex set $K \subset \mathbb{R}^{n}$ if and only if it can be expressed in the form (2.1), where $e \in$ bar $K \backslash$ nor $K$ and $\gamma=\sup \{x \cdot e: x \in K\}$.

We will say that a closed halfspace $V \subset \mathbb{R}^{n}$ bounds a convex set $K \subset \mathbb{R}^{n}$ provided $K \subset V$. Furthermore, if $H$ denotes the boundary hyperplane of $V$, then $V$ supports (properly supports) $K$ is $H$ supports (properly supports) $K$. Also, $V$ strictly (strongly) bounds $K$ is $H$ disjoint (strongly disjoint) from $K$. Various results on the existence or on description of different types of bounding halfspaces can be routinely derived from the above assertions on bounding hyperplanes. The results below deal with various representations of the closure of a given proper convex set $K \subset \mathbb{R}^{n}$ as intersections of bounding halfspaces (see [76, Chapter 9]). 
14. $\mathrm{cl} K$ is the intersection of all closed halfspaces bounding (supporting, strictly, or strongly bounding) $K$.

15. If $K$ is not a plane, $X$ is a dense subset of $\operatorname{rbd} K$, and $\mathcal{V}(X)$ is the family of all closed halfspaces $V$ each properly supporting $K$ at a point from $X$, then

$$
\operatorname{cl} K=\cap(V: V \in \mathcal{V}(X)) \quad \text { and } \quad \operatorname{rint} K=\cap(\operatorname{int} V: V \in \mathcal{V}(X)) .
$$

16. If $E$ is a dense subset of the normal cone nor $K$, then $\mathrm{cl} K$ is the intersection of a countable family $\mathcal{F}$ of closed halfspaces of the form

$$
V_{e}(\gamma)=\left\{x \in \mathbb{R}^{n}: x \cdot e \leq \gamma\right\}, \quad \text { where } e \in E, \gamma \geq \sup \{u \cdot e: u \in K\}
$$

17. If $K$ is line-free, then $\operatorname{cl} K=\cap(V: V \in \mathcal{G})$, where $\mathcal{G}$ denotes the family of all closed halfspaces supporting $\mathrm{cl} K$ at its exposed points.

\section{Separation of Convex Sets}

5.1. Classification of Separating Hyperplanes Various results on hyperplane separation of convex sets are usually formulated in the following terms. If $K_{1}$ and $K_{2}$ are convex sets in $\mathbb{R}^{n}$ and $H \subset \mathbb{R}^{n}$ is a hyperplane, then we say that

1. $H$ separates $K_{1}$ and $K_{2}$ if $K_{1}$ and $K_{2}$ lie in the opposite closed halfspaces determined by $H$ (possibly, $K_{1} \cup K_{2} \subset H$ ).

2. $H$ properly separates $K_{1}$ and $K_{2}$ if $H$ separates $K_{1}$ and $K_{2}$ such that $K_{1} \cup K_{2} \not \subset H$.

3. $H$ definitely separates $K_{1}$ and $K_{2}$ if $H$ separates $K_{1}$ and $K_{2}$ such that $K_{1} \not \subset H$ and $K_{2} \not \subset H$.

4. $H$ strictly separates $K_{1}$ and $K_{2}$ if $H$ separates $K_{1}$ and $K_{2}$ such that both sets are disjoint from $H$ (equivalently, $K_{1}$ and $K_{2}$ lie in the opposite open halfspaces determined by $H$ ).

5. $H$ strongly separates $K_{1}$ and $K_{2}$ if $H$ separates suitable open neighborhoods $U_{\rho}\left(K_{1}\right)$ and $U_{\rho}\left(K_{2}\right)$. 


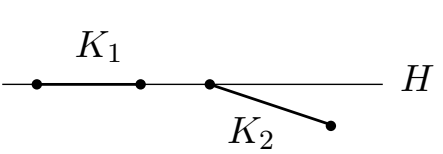

(b) Proper separation

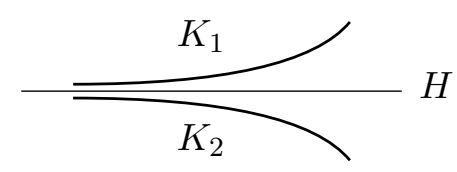

(d) Strict separation

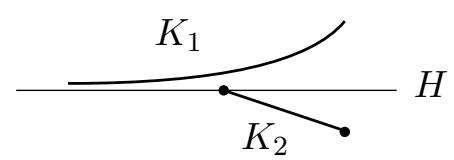

(c) Definite separation

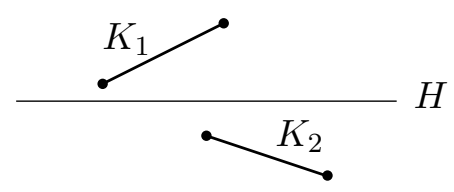

(e) Strong separation

Figure 12: Types of hyperplane separation of convex sets.

The above terminology gradually evolved in time: the term proper separation is due to Rockafellar [72, p. 95], definite separation is called real by Bair and Jongmans [3], strict separation and strong separation are due to Klee [51.

The following obvious assertions provide analytical equivalences of the above definitions on separation of convex sets $K_{1}$ and $K_{2}$ in $\mathbb{R}^{n}$ by a hyperplane $H \subset \mathbb{R}^{n}$ of the form 2.1):

1! $H$ separates $K_{1}$ and $K_{2}$ if and only if $e$ and $\gamma$ can be chosen such that

$$
\sup \left\{x \cdot e: x \in K_{1}\right\} \leq \gamma \leq \inf \left\{x \cdot e: x \in K_{2}\right\} .
$$

2! $H$ properly separates $K_{1}$ and $K_{2}$ if and only if $e$ and $\gamma$ can be chosen to satisfy both inequalities (5.1) and

$$
\inf \left\{x \cdot e: x \in K_{1}\right\}<\sup \left\{x \cdot e: x \in K_{2}\right\} .
$$

3! $H$ definitely separates $K_{1}$ and $K_{2}$ if and only if $e$ and $\gamma$ can be chosen to satisfy both inequalities (5.1) and

$$
\inf \left\{x \cdot e: x \in K_{1}\right\}<\gamma<\sup \left\{x \cdot e: x \in K_{2}\right\} .
$$

4. $H$ strictly separates $K_{1}$ and $K_{2}$ if and only if $e$ and $\gamma$ can be chosen such that both conditions below are satisfied:

$$
\begin{aligned}
u \cdot e<\inf \left\{x \cdot e: x \in K_{2}\right\} & \text { for every } u \in K_{1}, \\
\sup \left\{x \cdot e: x \in K_{1}\right\}<u \cdot e & \text { for every } u \in K_{2} .
\end{aligned}
$$


5! $H$ strongly separates $K_{1}$ and $K_{2}$ if and only if $e$ and $\gamma$ can be chosen such that

$$
\sup \left\{x \cdot e: x \in K_{1}\right\}<\gamma<\inf \left\{x \cdot e: x \in K_{2}\right\}
$$

The above types of separation can be refined by using asymmetric conditions (see Klee [51] and [78] for further details): If convex sets $K_{1}$ and $K_{2}$ in $\mathbb{R}^{n}$ are separated by a hyperplane $H \subset \mathbb{R}^{n}$, then we say that

6. $H$ properly separates $K_{1}$ from $K_{2}$ provided $K_{1} \not \subset H$.

7. $H$ strictly separates $K_{1}$ from $K_{2}$ provided $K_{1}$ is disjoint from $H$.

The relation between symmetric and asymmetric types of separation is described in the following theorem.

Theorem 5.1. ([76, Theorem 10.6]) If $K_{1}$ and $K_{2}$ are convex sets and $H_{1}$ and $H_{2}$ are hyperplanes such that $H_{i}$ properly (strictly) separates $K_{i}$ from $K_{3-i}, i=1,2$, then there is a hyperplane containing $H_{1} \cap H_{2}$ and properly (strictly) separating $K_{1}$ and $K_{2}$.

The results below describe the hyperplanes which separate a pair of convex sets and have a given direction of normals or contain a given point. An initial step towards description of separating hyperplanes with a given direction of normals consists in reduction to the case of a single convex set.

Theorem 5.2. ([76, Theorem 10.7]) Let $K_{1}$ and $K_{2}$ be convex sets in $\mathbb{R}^{n}$, and $H$ be a hyperplane of the form (2.1). Then the assertions below hold.

(a) A translate of $H$ separates (properly separates) $K_{1}$ and $K_{2}$ if and only if the subspace (2.2) bounds (properly bounds) $K_{1}-K_{2}$.

(b) A translate of $H$ strictly separates at least one of the sets $K_{1}$ and $K_{2}$ from the other if and only if the subspace (2.2) strictly bounds $K_{1}-K_{2}$.

(c) A translate of $H$ strongly separates $K_{1}$ and $K_{2}$ if and only if the subspace (2.2) strongly bounds $K_{1}-K_{2}$.

A combination of Theorems $4.8-4.10$ and Theorem 5.2 implies the following assertions.

Theorem 5.3. ([78, 82]) Given convex sets $K_{1}$ and $K_{2}$ in $\mathbb{R}^{n}$, the following assertions hold. 
(a) There is a hyperplane separating $K_{1}$ and $K_{2}$ if and only if any of the following equivalent conditions holds:

$$
\operatorname{int} K_{1} \cap \operatorname{int} K_{2}=\varnothing, \quad o \notin \operatorname{int}\left(K_{1}-K_{2}\right), \quad\left(K_{1}-K_{2}\right)^{\circ} \neq\{o\} .
$$

Furthermore, a translate of a hyperplane $H \subset \mathbb{R}^{n}$ separates $K_{1}$ and $K_{2}$ if and only if $H$ can be expressed in the form (2.1), where $e \in\left(K_{1}-K_{2}\right)^{\circ} \backslash\{o\}$.

(b) There is a hyperplane properly separating $K_{1}$ and $K_{2}$ if and only if any of the following equivalent conditions holds:

$$
\begin{aligned}
& \operatorname{rint} K_{1} \cap \operatorname{rint} K_{2}=\varnothing, \quad o \notin \operatorname{rint}\left(K_{1}-K_{2}\right), \\
& \left(K_{1}-K_{2}\right)^{\circ} \backslash \operatorname{lin}\left(K_{1}-K_{2}\right)^{\circ} \neq \varnothing .
\end{aligned}
$$

Furthermore, a translate of a hyperplane $H \subset \mathbb{R}^{n}$ properly separates $K_{1}$ and $K_{2}$ if and only if $H$ can be expressed in the form (2.1), where $e \in\left(K_{1}-K_{2}\right)^{\circ} \backslash \operatorname{lin}\left(K_{1}-K_{2}\right)^{\circ}$.

(c) There is a hyperplane definitely separating $K_{1}$ and $K_{2}$ if and only if either $o \notin \operatorname{cl}\left(K_{1}-K_{2}\right)$ or

$$
o \in \operatorname{cl}\left(K_{1}-K_{2}\right) \quad \text { and } \quad\left(K_{1}-K_{2}\right)^{\circ} \backslash\left(\operatorname{ort} K_{1} \cup \operatorname{ort} K_{2}\right) \neq \varnothing .
$$

Furthermore, a translate of a hyperplane $H \subset \mathbb{R}^{n}$ definitely separates $K_{1}$ and $K_{2}$ if and only if $H$ can be expressed in the form (2.1) such that one of the following conditions is satisfied:

(i) $o \notin \operatorname{cl}\left(K_{1}-K_{2}\right)$ and

$$
e \in \operatorname{rint}\left(K_{1}-K_{2}\right)^{\circ} \cup\left(\operatorname{rbd}\left(K_{1}-K_{2}\right)^{\circ} \backslash\left(\operatorname{ort} K_{1} \cup \operatorname{ort} K_{2}\right)\right),
$$

(ii) $o \in \operatorname{cl}\left(K_{1}-K_{2}\right)$ and $e \in\left(K_{1}-K_{2}\right)^{\circ} \backslash\left(\operatorname{ort} K_{1} \cup \operatorname{ort} K_{2}\right)$.

(d) There exists some hyperplane strongly separating $K_{1}$ and $K_{2}$ if and only if $o \notin \mathrm{cl}\left(K_{1}-K_{2}\right)$. Furthermore, if $o \notin \mathrm{cl}\left(K_{1}-K_{2}\right)$ and $e \in \operatorname{rint}\left(K_{1}-K_{2}\right)^{\circ}$, then a suitable translate of a hyperplane of the form 2.1) strongly separates $K_{1}$ and $K_{2}$.

A variation of Theorem 5.3 allows us to describe all hyperplanes which separate a pair of convex sets and contain a given point of $\mathbb{R}^{n}$. This description uses the following auxiliary lemma. 
Lemma 5.4. ([82]) Convex sets $K_{1}$ and $K_{2}$ in $\mathbb{R}^{n}$ are separated (properly separated) by a hyperplane $H \subset \mathbb{R}^{n}$ through a given point $z \in \mathbb{R}^{n}$ if and only if the generated cones $C_{z}\left(K_{1}\right)$ and $C_{z}\left(K_{2}\right)$ are separated (properly separated) by $H$.

Theorem 5.5. ([82]) Given convex sets $K_{1}$ and $K_{2}$ in $\mathbb{R}^{n}$ and a point $z \in \mathbb{R}^{n}$, let $D_{1}=C_{z}\left(K_{1}\right)-z, D_{2}=C_{z}\left(K_{2}\right)-z$. The assertions below hold.

(a) There is a hyperplane through $z$ separating $K_{1}$ and $K_{2}$ if and only if the cones $D_{1}$ and $D_{2}$ satisfy any of the following equivalent conditions:

$$
o \notin \operatorname{int}\left(D_{1}-D_{2}\right), \quad\left(D_{1}-D_{2}\right)^{\circ} \neq\{o\}, \quad D_{1}^{\circ} \cap\left(-D_{2}^{\circ}\right) \neq\{o\} .
$$

(b) There is a hyperplane through $z$ properly separating $K_{1}$ and $K_{2}$ if and only if the cones $D_{1}$ and $D_{2}$ satisfy any of the following equivalent conditions:

$$
\begin{gathered}
o \notin \operatorname{rint}\left(D_{1}-D_{2}\right), \quad\left(D_{1}-D_{2}\right)^{\circ} \text { is not a subspace, } \\
D_{1}^{\circ} \cap\left(-D_{2}^{\circ}\right) \text { is not a subspace. }
\end{gathered}
$$

5.2. Geometric Conditions on Hyperplane Separation Theorem 5.3 provides a unified description of all hyperplanes separating convex sets $K_{1}$ and $K_{2}$ in $\mathbb{R}^{n}$, which is formulated in terms of the polar cone $\left(K_{1}-K_{2}\right)^{\circ}$. Some other types of geometric conditions that guaranty the existence of a desired type of separation are given below.

Proper separation. The condition $\operatorname{rint} K_{1} \cap \operatorname{rint} K_{2}=\varnothing$ was already mentioned in Theorem 5.3. It was obtained in various forms of generality by Fenchel [30, p. 48], Klee [46], and Rockafellar [72, Theorem 11.3].

A related result on asymmetric type of proper separation is due to Rockafellar [72, Theorem 20.2]: Given convex sets $K_{1}$ and $K_{2}$ in $\mathbb{R}^{n}$ such that $K_{2}$ is polyhedral, there is a hyperplane properly separating $K_{1}$ from $K_{2}$ if and only if $\operatorname{rint} K_{1} \cap K_{2}=\varnothing$.

We observe that the latter assertion does not hold if the set $K_{2}$ is not polyhedral. For instance, if $K_{1}$ and $K_{2}$ are planar circular disks in $\mathbb{R}^{3}$ given by

$$
\begin{aligned}
& K_{1}=\left\{(x, y, 0): x^{2}+(y-1)^{2} \leq 1\right\}, \\
& K_{2}=\left\{(0, y, z): y^{2}+(z-1)^{2} \leq 1\right\},
\end{aligned}
$$

then $\operatorname{rint} K_{1} \cap K_{2}=\varnothing$, while $K_{1}$ is not properly separated from $K_{2}$. 
Strict separation. The only known geometric result on strict separation of convex sets is attributed to Klee [47]: If none of the disjoint closed convex sets $K_{1}$ and $K_{2}$ in $\mathbb{R}^{n}$ is a plane, and none of the sets rbd $K_{1}$ and $\operatorname{rbd} K_{2}$ contains a halfline, then $K_{1}$ and $K_{2}$ are strictly separated by a hyperplane.

Strong separation. An obvious continuity argument shows that if convex sets $K_{1}$ and $K_{2}$ are strongly separated by a hyperplane $H$, then there is a slab of positive width which separates $K_{1}$ and $K_{2}$ and whose boundary hyperplanes are parallel to $H$. A natural question here is to determine the maximum possible width of such a slab. The answer to this question was given by Dax [20] (see also [76, Theorem 10.20]).

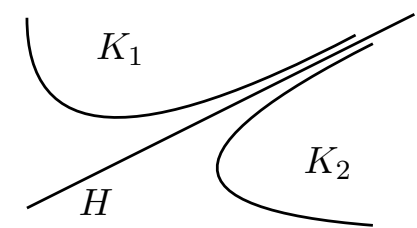

Figure 13: Strict separation of $K_{1}$ and $K_{2}$.

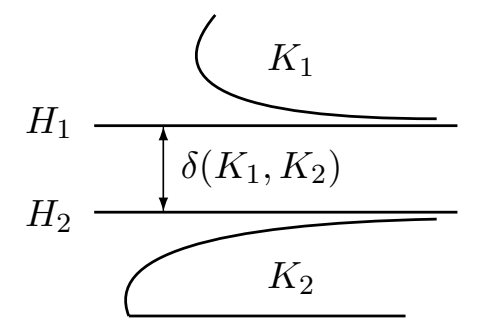

Figure 14: Separation of $K_{1}$ and $K_{2}$ by a slab of maximum width.

TheOREM 5.6. ([20]) If convex sets $K_{1}$ and $K_{2}$ in $\mathbb{R}^{n}$ are strongly disjoint (that is, $\delta\left(K_{1}, K_{2}\right)>0$ ), then there is a unique pair of parallel hyperplanes $H_{1}$ and $H_{2}$ in $\mathbb{R}^{n}$, both separating $K_{1}$ and $K_{2}$ and satisfying the condition $\delta\left(H_{1}, H_{2}\right)=\delta\left(K_{1}, K_{2}\right)$.

The above equality $\delta\left(H_{1}, H_{2}\right)=\delta\left(K_{1}, K_{2}\right)$, without specifying the uniqueness of the pair $\left\{H_{1}, H_{2}\right\}$, was obtained later by Gabidullina [32] for the case when at least one of the sets $K_{1}$ and $K_{2}$ is compact.

A similar question on strong separation of convex sets $K_{1}$ and $K_{2}$ concerns the existence of a pair of nearest points $z_{1} \in \operatorname{cl} K_{1}$ and $z_{2} \in \operatorname{cl} K_{2}$. A simple 
geometric argument shows that in this case the hyperplanes through $z_{1}$ and $z_{2}$ orthogonal to $\left[z_{1}, z_{2}\right]$ form a slab of maximum width separating $K_{1}$ and $K_{2}$. A sufficient condition for the existence of such a pair $\left\{z_{1}, z_{2}\right\}$ can be found in [76].

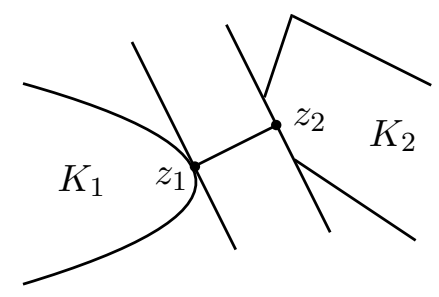

Figure 15: A nearest pair of points in $K_{1}$ and $K_{2}$.

Theorem 5.7. ([76, Theorem 10.24]) If convex sets $K_{1}$ and $K_{2}$ in $\mathbb{R}^{n}$ satisfy the condition $\operatorname{rec}\left(\operatorname{cl} K_{1}\right) \cap \operatorname{rec}\left(\operatorname{cl} K_{2}\right)=\{o\}$, then $\delta\left(K_{1}, K_{2}\right)=\left\|z_{1}-z_{2}\right\|$ for suitable points $z_{1} \in \operatorname{cl} K_{1}$ and $z_{2} \in \mathrm{cl} K_{2}$. In particular, a nearest pair $\left\{z_{1}, z_{2}\right\}$ exists provided at least one of the sets $K_{1}$ and $K_{2}$ is bounded.

A useful result on strong separation of convex sets was obtained by De Wilde [21].

ThEOREM 5.8. ([21]) If $K_{1}$ and $K_{2}$ are disjoint closed convex sets in $\mathbb{R}^{n}$, then the following conditions are equivalent:

(a) Both $K_{1}$ and $K_{2}$ are line-free and $\operatorname{rec} K_{1} \cap \operatorname{rec} K_{2}=\{o\}$.

(b) There are parallel disjoint hyperplanes $H_{1}$ and $H_{2}$ both separating $K_{1}$ and $K_{2}$ such that $H_{i} \cap K_{i}$ is an exposed point of $K_{i}, i=1,2$.

Analysis of the proof of Theorem 5.8 shows that the exposed points $H_{1} \cap K_{1}$ and $H_{2} \cap K_{2}$ are not necessarily the nearest. Nevertheless, $H_{1}$ and $H_{2}$ may be chosen to satisfy the condition $\delta\left(H_{1}, H_{2}\right)>\delta\left(K_{1}, K_{2}\right)-\varepsilon$ for any given scalar $\varepsilon>0$.

Maximal separation. Klee [50] obtained various results regarding strict and strong separation of convex sets by hyperplanes. Given a pair $\{\mathcal{F}, \mathcal{G}\}$ of nonempty families of closed convex sets in $\mathbb{R}^{n}$, we say that $\mathcal{F}$ is maximal with respect to a certain type $S$ of separation provided it satisfies the following conditions:

1. The sets $F$ and $G$ are $S$-separated whenever $F \in \mathcal{F}$ and $G \in \mathcal{G}$, with $F \cap G=\varnothing$. 
2. For every $F \in \mathcal{F}$, there is $G \in \mathcal{G}$ such that $F \cap G=\varnothing$.

3. For every closed convex set $F \notin \mathcal{F}$, there is $G \in \mathcal{G}$ such that $F \cap G=\varnothing$ but $F$ and $G$ are not $S$-separated.

Following Gale and Klee [33, we say that a closed convex set $K \subset \mathbb{R}^{n}$ is continuous provided $K$ admits no boundary halfline and no line asymptote. Given disjoint closed convex sets $F$ and $G$ in $\mathbb{R}^{n}$, the assertions below hold (see [50]).

4. Each of the following conditions implies that $F$ and $G$ are strictly separated and represents a maximal theorem for strict separation:

(a) $F$ is continuous,

(b) neither $F$ nor $G$ admits a line asymptote,

(c) neither $F$ nor $G$ has a boundary halfline.

5. Each of the following conditions implies that $F$ and $G$ are strongly separated and represents a maximal theorem for strong separation:

(d) $F$ is continuous,

(e) neither $F$ nor $G$ admits a line asymptote.

An extensive development and generalization of Klee's results on maximal separation is given in the book of Fajardo, Goberna, Rodríguez, and VicentePérez [27]. Theorem 1.2 and Theorem 1.3 from this book give a comprehensive list of various maximal separation assertions for the case of evenly convex sets. (According to Fenchel [29], a convex set in $\mathbb{R}^{n}$ is called evenly convex if it is the intersection of a family of open halfspaces. It is easy to see that every proper closed convex sets is evenly convex.)

5.3. Sharp Separation of Convex Cones If convex cones $C_{1}$ and $C_{2}$ with a common apex in $\mathbb{R}^{n}$ are separated by a hyperplane $H \subset \mathbb{R}^{n}$, then $H$ supports both cones $\operatorname{cl} C_{1}$ and $\operatorname{cl} C_{2}$. Consequently, ap $\left(\operatorname{cl} C_{1}\right) \cup \operatorname{ap}\left(\operatorname{cl} C_{2}\right) \subset H$. In this regard, we will say that $H$ sharply separates $C_{1}$ from $C_{2}$ provided $H \cap \operatorname{cl} C_{1}=\operatorname{ap}\left(\operatorname{cl} C_{1}\right)$. Similarly, $H$ sharply separates $C_{1}$ and $C_{2}$ if

$$
H \cap\left(\operatorname{cl} C_{1}\right)=\operatorname{ap}\left(\operatorname{cl} C_{1}\right) \quad \text { and } \quad H \cap \operatorname{cl} C_{2}=\operatorname{ap}\left(\operatorname{cl} C_{2}\right) .
$$

The next two theorems give criteria for sharp separation of cones in terms of their polar cones. 
THEOREM 5.9. ([78]) If $C_{1}$ and $C_{2}$ are convex cones in $\mathbb{R}^{n}$ with a common apex $a \in \mathbb{R}^{n}$, then the following conditions are equivalent.

(a) $C_{1}$ is sharply separated from $C_{2}$.

(b) The set $E=\operatorname{rint}\left(C_{1}-a\right)^{\circ} \cap\left(a-C_{2}\right)^{\circ}$ has positive dimension.

Theorem 5.10. ([74, 78]) If $C_{1}$ and $C_{2}$ are convex cones in $\mathbb{R}^{n}$ with a common apex $a \in \mathbb{R}^{n}$, then the following conditions are equivalent.

(a) $C_{1}$ and $C_{2}$ are sharply separated.

(b) Each of the cones $C_{1}$ and $C_{2}$ is sharply separated from the other.

(c) The set $D=\operatorname{rint}\left(C_{1}-a\right)^{\circ} \cap \operatorname{rint}\left(a-C_{2}\right)^{\circ}$ has positive dimension.

Analysis of the proof of Theorem 5.10 reveals a simple corollary: If $C_{1}$ is not a plane and is sharply separated from $C_{2}$, then $C_{1}$ is properly separated from $C_{2}$. The converse assertion is not true. For instance, in $\mathbb{R}^{2}$, the cone $C_{1}=\{(x, 0): x \in \mathbb{R}\}$ is separated sharply but not properly from the cone $C_{2}=\{(x, y): 0 \leq x, 0 \leq y \leq x\}$, while $C_{2}$ is separated properly but not sharply from $C_{1}$ (see Figure 16).

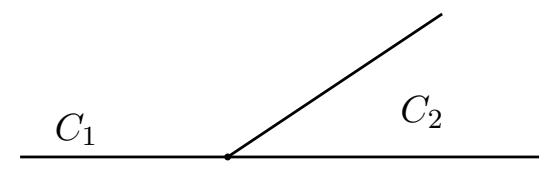

Figure 16: Proper but not sharp separation of cones $C_{1}$ and $C_{2}$.

A geometric criterion for sharp separation of convex cones is given in the following theorem.

Theorem 5.11. ([74]) Let $C_{1}$ and $C_{2}$ be convex cones in $\mathbb{R}^{n}$ with a common apex $a \in \mathbb{R}^{n}$. The conditions below are equivalent.

(a) $C_{1}$ and $C_{2}$ are sharply separated by a hyperplane.

(b) $\operatorname{cl} C_{1} \cap \operatorname{cl} C_{2}=\operatorname{ap}\left(\operatorname{cl} C_{1}\right) \cap \operatorname{ap}\left(\operatorname{cl} C_{2}\right)$ and at least one of the cases below holds:

(i) $\operatorname{dim}\left(C_{1} \cup C_{2}\right) \leqslant n-1$,

(ii) at least one of the cones $C_{1}$ and $C_{2}$ is not a plane. 
In terms of continuous linear functionals on a linear topological space, Theorem 5.11, formulated for the case of closed convex cones with a common apex $o$, was proved earlier by Klee [46] under the assumption ap $C_{1} \cap$ ap $C_{2}=\{o\}$, and by Bair and Gwinner [2] under the condition that $\operatorname{ap} C_{1} \cap \operatorname{ap} C_{2}$ is a subspace.

5.4. Penumbras and Separation Following Rockafellar [72, p. 22], we recall that the penumbra of a convex set $K_{1}$ with respect to another convex set $K_{2}$, denoted below $P\left(K_{1}, K_{2}\right)$, is defined by

$$
\begin{aligned}
P\left(K_{1}, K_{2}\right) & =\cup\left(\mu K_{1}+(1-\mu) K_{2}: \mu \geq 1\right) \\
& =\left\{\mu x_{1}+(1-\mu) x_{2}: \mu \geq 1, x_{1} \in K_{1}, x_{2} \in K_{2}\right\} .
\end{aligned}
$$

Geometrically, $P\left(K_{1}, K_{2}\right)$ is the union of all closed halflines initiated at the points of $K_{1}$ in the directions of vectors from $K_{1}-K_{2}$ (see Fig. 17). It is possible to show (see [80]) that both sets $P\left(K_{1}, K_{2}\right)$ and $P\left(K_{2}, K_{1}\right)$ are convex and contain $K_{1}$ and $K_{2}$, respectively. The following theorem illustrates the role of penumbras in separation of convex sets.

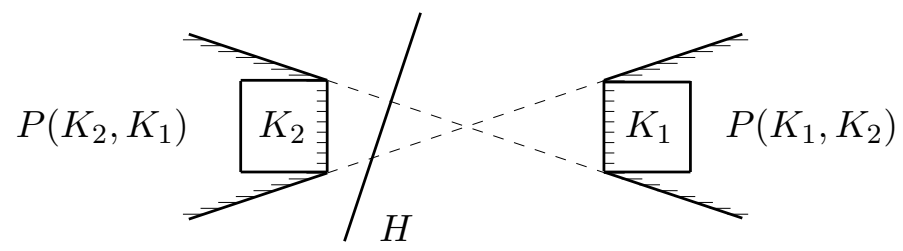

Figure 17: Illustration to Theorem 5.12 .

Theorem 5.12. ([80]) Let $K_{1}$ and $K_{2}$ be convex sets in $\mathbb{R}^{n}$. A hyperplane $H \subset \mathbb{R}^{n}$ separates (respectively, properly, strictly, or strongly) $K_{1}$ and $K_{2}$ if and only if it separates (respectively, nontrivially, strictly, or strongly) the sets $P\left(K_{1}, K_{2}\right)$ and $P\left(K_{2}, K_{1}\right)$.

Given convex sets $K_{1}$ and $K_{2}$ in $\mathbb{R}^{n}$, denote by $\mathcal{H}_{1}\left(K_{1}, K_{2}\right)$ (respectively, by $\mathcal{H}_{2}\left(K_{1}, K_{2}\right)$ and $\mathcal{H}_{3}\left(K_{1}, K_{2}\right)$ ) the family of all hyperplanes properly (respectively, strictly and strongly) separating $K_{1}$ and $K_{2}$. Also, let

$$
E_{i}\left(K_{1}, K_{2}\right)=\cup\left(H: H \in \mathcal{H}_{i}\right), \quad i=1,2,3 .
$$


TheOREm 5.13. ([80]) If convex sets $K_{1}$ and $K_{2}$ in $\mathbb{R}^{n}$ satisfy the condition $\operatorname{rint} K_{1} \cap \operatorname{rint} K_{2}=\varnothing$, then

$$
E_{1}\left(K_{1}, K_{2}\right)=\mathbb{R}^{n} \backslash\left(\operatorname{rint} P\left(K_{1}, K_{2}\right) \cup \operatorname{rint} P\left(K_{2}, K_{1}\right)\right) .
$$

Furthermore, a hyperplane $H \subset \mathbb{R}^{n}$ properly separates $K_{1}$ and $K_{2}$ if and only if $H \subset E_{1}\left(K_{1}, K_{2}\right)$ and $H \cap$ aff $\left(K_{1} \cup K_{2}\right) \neq \varnothing$.

Corollary 5.14. ([80]) If convex sets $K_{1}$ and $K_{2}$ in $\mathbb{R}^{n}$ satisfy the condition $\mathrm{cl} K_{1} \cap \operatorname{cl} K_{2}=\varnothing$, then $E_{2}\left(K_{1}, K_{2}\right) \subset F_{2}\left(K_{1}, K_{2}\right)$, where

$$
\left.F_{2}\left(K_{1}, K_{2}\right)=\mathbb{R}^{n} \backslash\left(P\left(\operatorname{cl} K_{1}, \operatorname{cl} K_{2}\right) \cup P\left(\operatorname{cl} K_{2}, \operatorname{cl} K_{1}\right)\right)\right) .
$$

Furthermore, a hyperplane $H \subset \mathbb{R}^{n}$ strictly separates $\mathrm{cl} K_{1}$ and $\mathrm{cl} K_{2}$ if and only if $H \subset F_{2}\left(K_{1}, K_{2}\right)$ and $H \cap$ aff $\left(K_{1} \cup K_{2}\right) \neq \varnothing$.

The inclusion $E_{2}\left(K_{1}, K_{2}\right) \subset F_{2}\left(K_{1}, K_{2}\right)$ in Corollary 5.14 may be proper. Indeed, consider the closed convex sets

$$
K_{1}=\{(x, 1): 0 \leq x \leq 1\} \quad \text { and } \quad K_{2}=\{(x, 0): x \in \mathbb{R}\} .
$$

Then $E_{2}\left(K_{1}, K_{2}\right)=\{(x, y): 0<y<1\}$, while

$$
F_{2}\left(K_{1}, K_{2}\right)=E_{2}\left(K_{1}, K_{2}\right) \cup\{(x, 1): x<0\} \cup\{(x, 1): x>1\} .
$$

THEOREM 5.15. ([80]) If convex sets $K_{1}$ and $K_{2}$ in $\mathbb{R}^{n}$ are strongly disjoint, then

$$
E_{3}\left(K_{1}, K_{2}\right)=\mathbb{R}^{n} \backslash\left(\operatorname{cl} P\left(K_{1}, K_{2}\right) \cup \operatorname{cl} P\left(K_{2}, K_{1}\right)\right) .
$$

The following assertions from [80] relate various properties of penumbras to some known classes of convex sets in $\mathbb{R}^{n}$.

1. If $K_{1}$ is compact, then $P\left(K_{1}, K_{2}\right)$ is an M-predecomposable set.

2. If both $K_{1}$ and $K_{2}$ are compact and $K_{1} \cap K_{2}=\varnothing$, then $P\left(K_{1}, K_{2}\right)$ is an M-decomposable set.

3. If both $K_{1}$ and $K_{2}$ are polyhedra, then $\operatorname{cl} P\left(K_{1}, K_{2}\right)$ is a polyhedron.

4. If both $K_{1}$ and $K_{2}$ are polytopes, then $P\left(K_{1}, K_{2}\right)$ is a polyhedron. 
5.5. Hemispaces The following concept was introduced by Motzkin 66, Lecture III] in three dimensions, and, independently, by Hammer [39] in vector spaces of any dimension: Given a point $v \in \mathbb{R}^{n}$, any maximal (under inclusion) convex subset of $\mathbb{R}^{n} \backslash\{v\}$, denoted $S_{v}$, is called a semispace of $\mathbb{R}^{n}$ at $v$ (in [43] and [53] these sets are called hypercones). The next properties of semispaces can be easily obtained (see [39, 43, 66]).

1. For a semispace $S_{v} \subset \mathbb{R}^{n}$, both sets $S_{v}$ and $\mathbb{R}^{n} \backslash S_{v}$ are convex cones with apex $v$.

2. For a convex set $K \subset \mathbb{R}^{n} \backslash\{v\}$, there is a semispace $S_{v} \subset \mathbb{R}^{n}$ containing $K$.

3. If $C \subset \mathbb{R}^{n}$ is a convex cone with improper apex $v \in \mathbb{R}^{n}$ and $B \subset \mathbb{R}^{n}$ is a convex set missing $v$ and disjoint from $C$, then there is a semispace $S_{v} \subset \mathbb{R}^{n}$ containing $C$ and disjoint from $B$ (Jamison [43] for the case $v=o)$.

Additional properties of semispaces in vector spaces of any dimension can be found in the papers $[22,43,48,56,65]$.

Theorem 5.16. ([39]) The family of all semispaces of $\mathbb{R}^{n}$ is the smallest among all families $\mathcal{F}$ of convex sets in $\mathbb{R}^{n}$ satisfying the following condition: every proper convex set $K \subset \mathbb{R}^{n}$ is the intersection of some elements from $\mathcal{F}$.

The structure of semispaces can be described in different ways. The first one, briefly mentioned by Hammer [40] (see the books [58, Satz 1.10], and [76, Theorem 10.32] for complete proofs), uses a nested family of planes

$$
\{v\}=L_{0} \subset L_{1} \subset \cdots \subset L_{n-1} \subset L_{n}=\mathbb{R}^{n}, \quad \operatorname{dim} L_{i}=i, 0 \leq i \leq n,
$$

and their halfplanes $E_{1}, \ldots, E_{n}$, where $E_{i}$ an open halfplane of $L_{i}$ determined by $L_{i-1}, 1 \leq i \leq n$.

Theorem 5.17. ([40]) If $S_{v} \subset \mathbb{R}^{n}$ is a semispace at $v \in \mathbb{R}^{n}$, then there is a nested sequence of planes of the form (5.6) and a respective sequence of open halfplanes $E_{1}, \ldots, E_{n}$ such that $S_{v}=E_{1} \cup \cdots \cup E_{n}$. Conversely, any set of the form $E_{1} \cup \cdots \cup E_{n}$ is a semispace at $v$.

Another way (given by Hammer [39, 40] without proof) is based on the choice of a suitable basis for $\mathbb{R}^{n}$.

Theorem 5.18. ([39, 40]) If $S_{v} \subset \mathbb{R}^{n}$ is a semispace at $v \in \mathbb{R}^{n}$, then there is a basis $e_{1}, \ldots, e_{n}$ for $\mathbb{R}^{n}$ such that $S_{v}$ consists of all vectors of the form 
$v+\alpha_{1} e_{1}+\cdots+\alpha_{n} e_{n}$, where $\alpha_{1}^{2}+\cdots+\alpha_{n}^{2}>0$ and the first nonzero scalar in the sequence $\alpha_{1}, \ldots, \alpha_{n}$ is positive. Conversely, given any basis $e_{1}, \ldots, e_{n}$ for $\mathbb{R}^{n}$, the set of described above vectors is a semispace at $v$.

The equivalence of description of semispaces in Theorem 5.17 and Theorem 5.18 follows from the simple geometric arguments:

4. If $e_{1}, \ldots, e_{n}$ is a basis for $\mathbb{R}^{n}$, then the open halfplanes $E_{i}$ from Theorem 5.17 can be chosen as

$$
E_{i}=v+\left\{\alpha_{n-i+1} e_{n-i+1}+\cdots+\alpha_{n} e_{n}: \alpha_{n-i+1}>0\right\}, \quad 1 \leq i \leq n .
$$

5. For any choice of planes (5.6) and of respective halfplanes $E_{1}, \ldots, E_{n}$, nonzero vectors

$$
e_{i} \in\left(E_{n-i+1}-v\right) \backslash\left(L_{n-i}-v\right), \quad 1 \leq i \leq n,
$$

form a basis for $\mathbb{R}^{n}$ such that the equalities (5.7) hold.

Independently, Martínez-Legaz [59] described a similar separation result, based on lexicographic ordering $\preceq$ of $\mathbb{R}^{n}$. We recall that for distinct vectors $x=\left(x_{1}, \ldots, x_{n}\right)$ and $y=\left(y_{1}, \ldots, y_{n}\right)$, one can write $x \prec y$ if $x_{i}<y_{i}$, with $i$ being the first index in $\{1, \ldots, n\}$ for which $x_{i} \neq y_{i}$; also, $x \preceq y$ if $x \prec y$ or $x=y$. In a standard way, a invertible $n \times n$ matrix $A$ is orthogonal if $A^{-1}=A^{T}$.

Theorem 5.19. ([59]) For a proper convex set $K \subset \mathbb{R}^{n}$ and a point $x_{0} \notin K$, there is an invertible (even orthogonal) $n \times n$ matrix $A$ and a vector $v \in \mathbb{R}^{n}$ such that $A x \prec v \preceq A x_{0}$ whenever $x \in K$.

Although Martínez-Legaz [59] made an observation that the sets from Theorem 5.19 are similar in their properties to semispaces, it was Singer [73] who proved the following assertion:

A set $M \subset \mathbb{R}^{n}$ is a semispace at $v$ if and only if there is an invertible matrix $n \times n$ matrix $A$ such that $M=\left\{x \in \mathbb{R}^{n}: A x \prec v\right\}$.

The result below is proved by Tukey [91] (the condition that the vector space $E$ should be normed is superfluous) and, independently, by Stone [85] (see Theorem 7 from Chapter 3).

Theorem 5.20. ([85, 91]) Any pair of disjoint convex sets $K_{1}$ and $K_{2}$ in a vector space $E$ can be separated by complementary convex sets $Q_{1}$ and $Q_{2}$ :

$$
K_{1} \subset Q_{1}, \quad K_{2} \subset Q_{2}, \quad Q_{1} \cup Q_{2}=E, \quad Q_{1} \cap Q_{2}=\varnothing .
$$


We observe that Theorem 5.20 cannot be extended to the case of more than two convex sets. For instance, the convex cones $C_{1}, C_{2}$, and $C_{3}$ in the plane, depicted in Figure 18, cannot be enlarged into pairwise disjoint (even pairwise non-overlapping) convex sets whose union is the entire plane.

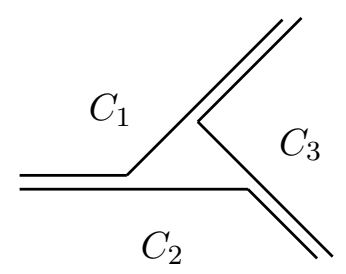

Figure 18: No convex extensions of cones $C_{1}, C_{2}$ and $C_{3}$ cover the whole plane.

The following results are similar to those from Theorem 5.20 .

6. If $C \subset \mathbb{R}^{n}$ is a convex cone with apex o such that $C \cap(-C)=\{o\}$, then there is a convex cone $C^{\prime}$ with apex $o$ satisfying the conditions $C \subset C^{\prime}$, $C^{\prime} \cap\left(-C^{\prime}\right)=\{o\}$, and $C^{\prime} \cup\left(-C^{\prime}\right)=\mathbb{R}^{n}$ (Ellis [26]).

7. Let $\mathcal{F}$ ba a commuting family of affine transformations in $\mathbb{R}^{n}$, and let $K_{1}$ and $K_{2}$ be disjoint convex sets both invariant with respect to transformations from $\mathcal{F}$. Then there are complementary $\mathcal{F}$-invariant convex sets $Q_{1}$ and $Q_{2}$ such that $K_{1} \subset Q_{1}$ and $K_{2} \subset Q_{2}$ (Páles [68]).

Following Jamison [42], we say that a proper convex subset $Q$ of $\mathbb{R}^{n}$ is a hemispace provided its complement $\mathbb{R}^{n} \backslash Q$ is a convex set. In [54] and [76], hemispaces are also called convex halfspaces. Various properties of hemispaces in vectors spaces of any dimension can be found in the papers [23, 42, 55] (see [36] for related material). A description of hemispaces in $\mathbb{R}^{n}$, similar to that of Theorem 5.18, was obtained by Lassak [54].

THEOREM 5.21. ([54]) If $Q$ and $Q^{\prime}$ are complementary hemispaces in $\mathbb{R}^{n}$, then there is a point $v \in \mathbb{R}^{n}$, a (orthogonal) basis $e_{1}, \ldots, e_{n}$ for $\mathbb{R}^{n}$, and an integer $r \geq 1$ such that one of the sets $Q$ and $Q^{\prime}$ consists of all vectors of the form $v+\alpha_{r} e_{r}+\cdots+\alpha_{n} e_{n}$, where $\alpha_{r}^{2}+\cdots+\alpha_{n}^{2}>0$ and the first nonzero scalar in the sequence $\alpha_{r}, \ldots, \alpha_{n}$ is positive. Conversely, for any choice of a point $v \in \mathbb{R}^{n}$, a basis $e_{1}, \ldots, e_{n}$ for $\mathbb{R}^{n}$, and an integer $r \geq 1$, the sets of vectors described above is a hemispace in $\mathbb{R}^{n}$. 
The next theorem shows that the above description of complementary hemispaces can be reformulated in terms of nested sequences of planes (5.6) and of their halfplanes $E_{1}, \ldots, E_{n}$.

Theorem 5.22. ([76, Theorem 10.28]) If $Q$ and $Q^{\prime}$ are complementary hemispaces in $\mathbb{R}^{n}$, then there is a sequence of planes of the form (5.6) and an integer $1 \leqslant r \leqslant n$ such that either $Q=F_{r}$ and $Q^{\prime}=F_{r}^{\prime}$, or $Q=F_{r}^{\prime}$ and $Q^{\prime}=F_{r}$, with

$$
F_{r}=E_{r} \cup \cdots \cup E_{n}, \quad F_{r}^{\prime}=L_{r-1} \cup E_{r}^{\prime} \cup \cdots \cup E_{n}^{\prime}, \quad 1 \leqslant r \leqslant n,
$$

where $E_{i}, E_{i}^{\prime}$ are complementary open halfplanes of $L_{i}$ determined by $L_{i-1}$.

Independently, Martínez-Legaz [59] defined a hemispace in $\mathbb{R}^{n}$ as the set of vectors $x \in \mathbb{R}^{n}$ satisfying the condition $A x \prec v$, where $A$ is an arbitrary (not necessarily invertible) $n \times n$ matrix and $v \in \mathbb{R}^{n}$. Later, Martínez-Legaz and Singer [60] proved that for any pair of disjoint convex sets $K_{1}$ and $K_{2}$ in $\mathbb{R}^{n}$, there exists an orthogonal $n \times n$ matrix $A$ such that $A x_{1} \prec A x_{2}$ for all $x_{1} \in K_{1}$ and $x_{2} \in K_{2}$.

The following description of hemispaces in terms of lexicographical order on $\overline{\mathbb{R}}^{n}$ is due to Martínez-Legaz and Singer 61 (here $\overline{\mathbb{R}}^{n}$ stands for the Cartesian product of $n$ extended lines $\overline{\mathbb{R}}=[-\infty, \infty])$.

Theorem 5.23. (61]) A set $Q \subset \mathbb{R}^{n}$ is a hemispace if and only if there is an $n \times n$ orthogonal matrix $A$ and a point $v \in \overline{\mathbb{R}}^{n}$ such that either $Q=\left\{x \in \mathbb{R}^{n}: A x \prec v\right\}$ or $Q=\left\{x \in \mathbb{R}^{n}: A x \preceq v\right\}$.

\section{ACKNOWLEDGEMENTS}

The author is thankful to the referees for the careful reading and considered suggestions leading to a better presented paper.

\section{REFERENCES}

[1] A. Auslender, M. Teboulle, "Asymptotic Cones and Functions in Optimization and Variational Inequalities", Springer, New York, 2003.

[2] J. BAir, J. Gwinner, Sur la séparation vraie de cônes convexes, Arkiv Mat. 16 (1978), 207-212.

[3] J. Bair, F. Jongmans, La séparation vraie dans un espace vectoriel, Bull. Soc. Roy. Sci. Liège 41 (1972), 163-170.

[4] F. Bernstein, Über das Gaußsche Fehlergesetz, Math. Ann. 64 (1907), $417-448$. 
[5] F. Bernstein, Konvexe Kurven mit überall dichter Menge von Ecken, Arch. Math. Phys. 12 (1907), 285-286.

[6] L. Bieberbach, "Differentialgeometrie", Teubner, Leipzig, 1932.

[7] W. Blaschke, "Kreis und Kugel", Viet \& Co., Leipzig, 1916.

[8] T. Bonnesen, W. Fenchel, "Theorie der konvexen Körper", Springer, Berlin, 1934. English translation: "Theory of Convex Bodies", BCS Associates, Moscow, ID, 1987.

[9] T. Botts, Convex sets, Amer. Math. Monthly 49 (1942), 527-535.

[10] H. Brunn, Zur Theorie der Eigebiete, Arch. Math. Phys. 17 (1911), 289-300.

[11] H. Brunn, Fundamentalsatz von den Stützen eines Eigebietes, Math. Ann. 100 (1928), 634-637.

[12] H. BRUnN, Vom Normalenkegel der Zwischenebenen zweier getrennter Eikörper, S.-B. Bayer. Akad. der Wiss. Math.-Natur. Klasse, München (1930), $165-182$.

[13] Yu.D. Burago, V.A. Zalgaller, Sufficient conditions for convexity, J. Soviet Math. 16 (1978), 395-434.

[14] D.G. Caraballo, Convexity, local simplicity, and reduced boundaries of sets, J. Convex Anal. 18 (2011), 823-832.

[15] D.G. Caraballo, Reduced boundaries and convexity, Proc. Amer. Math. Soc. 141 (2013), 1775-1782.

[16] C. Carathéodory, Über den Variabilitätsbereich der Koefficienten von Potenzreihen, die gegebene Werte nicht annehmen, Math. Ann. 64 (1907), 95- 115 .

[17] C. Carathéodory, Über den Variabilitätsbereich der Fourierschen Konstanten von positiven harmonischen Funktionen, Rend. Circ. Mat. Palermo 32 (1911), $193-217$.

[18] S.N. Černikov, "Linear Inequalities" (Russian), Nauka, Moscow, 1968.

[19] G.B. DAntzig, "Linear Programming and Extensions", Princeton University Press, Princeton, NJ, 1963.

[20] A. Dax, The distance between two convex sets, Linear Algebra Appl. 416 (2006), $184-213$.

[21] M. De WiLde, Some properties of the exposed points of finite dimensional convex sets, J. Math. Anal. Appl. 99 (1984), 257-264.

[22] J.C. Dupin, G. Coquet, Caractérisations et propriétés des couples de convexes complémentaires, C. R. Acad. Sci. Paris Sér. A-B 276 (1973), A $273-\mathrm{A} 276$.

[23] J.C. Dupin, G. Coquet, Caractérisations et propriétés des couples de convexes complémentaires, C. R. Acad. Sci. Paris Sér. A-B 276 (1973), A $383-\mathrm{A} 386$.

[24] R. DuRIER, The Fermat-Weber problem and inner product spaces, J. Approx. Theory 78 (1994), 161-173.

[25] M. Eidelheit, Zur Theorie der konvexen Mengen in linearen normierten Räumen, Stud. Math. 6 (1936), 104-111. 
[26] J.W. ElLIS, A general set-separation theorem, Duke Math. J. 19 (1952), $417-421$.

[27] M.D. Fajardo, M.A. Goberna, M.M.L. Rodríguez, J. VicentePÉREZ, "Even Convexity and Optimization", Springer, 2020.

[28] J. Favard, Sur les corps convexes, J. Math. Pures Appl. 12 (1933), 219-282.

[29] W. Fenchel, A remark on convex sets and polarity, Comm. Sém. Math. Univ. Lund. Tome Suppl. (1952), 82-89.

[30] W. Fenchel, "Convex Cones, Sets, and Functions. Mimeographed Lecture Notes. Spring Term 1951", Princeton University, Princeton, NJ, 1953.

[31] M. FujIwara, Über die Anzahl der Kantenlinien einer geschlossenen konvexen Fläche, Tôhoku Math. J. 10 (1916), 164-166.

[32] Z.R. Gabidullina, A linear separability criterion for sets of Euclidean space, J. Optim. Theory Appl. 158 (2013), 145-171.

[33] D. Gale, V. KleE, Continuous convex sets, Math. Scand. 7 (1959), 379-391.

[34] H. GeRICKE, Über ein Konvexitätskriterium, Math. Z. 43 (1937), 110-112.

[35] M.A. Goberna, E. GonzÁlez, J.E. Martínez-Legaz, M.I. TODOROV, Motzkin decomposition of closed convex sets, J. Math. Anal. Appl. 364 (2010), 209-221.

[36] V.V. Gorokhovik, E.A. Semenkova, Step-linear functions in finitedimensional vector spaces. Definition, properties and their relation to halfspaces, (Russian) Dokl. Akad. Nauk Belarusi 41 (1997), 10-14.

[37] O. GüLER, "Foundations of Optimization", Springer, New York, 2010.

[38] B.P. HaAlmeijer, On convex regions, Nieuw Arch. Wisk. 12 (1917), $152-160$.

[39] P.C. Hammer, Maximal convex sets, Duke Math. J. 22 (1955), 103-106.

[40] P.C. Hammer, Semispaces and the topology of convexity, in "Convexity" (edited by V-L. Klee), Amer. Math. Soc., Providence, RI, 1963, 305-316.

[41] A.N. Iusem, J.E. Martínez-Legaz, M.I. Todorov, Motzkin predecomposable sets, J. Global Optim. 60 (2014), 635-647.

[42] R.E. JAmison, Some intersection and generation properties of convex sets, Compos. Math. 35 (1977), 147-161.

[43] R.E. Jamison, The space of maximal convex sets, Fund. Math. 111 (1981), $45-59$.

[44] J.L. Jensen, Sur les fonctions convexes et les inégalités entre les inégalités entre les valeurs moyeunes, Acta Math. 30 (1906), 175-193.

[45] S. KakeyA, On some properties of convex curves and surfaces, Tôhoku Math. J. 8 (1915), 218-221.

[46] V.L. KleE, Separation properties of convex cones, Proc. Amer. Math. Soc. 6 (1955), 313-318.

[47] V.L. KLEE, Strict separation of convex sets, Proc. Amer. Math. Soc. 7 (1956), $735-737$.

[48] V.L. KLEE, The structure of semispaces, Math. Scand. 4 (1956), 54-64. 
[49] V.L. KLEE, Asymptotes and projections of convex sets, Math. Scand. 8 (1960), $356-362$.

[50] V.L. KLEE, Maximal separation theorems for convex sets, Trans. Amer. Math. Soc. 134 (1968), 133-147.

[51] V.L. KLEE, Separation and support properties of convex sets-a survey, In: A. V. Balakrishnan (ed), "Control Theory and the Calculus of Variations", Academic Press, New York, 1969, pp. 235-303.

[52] V.L. KLEE, Sharper approximation of extreme points by far points, Arch. Math. (Basel) 60 (1993), 383-388.

[53] G. KӧтнE, "Topologische Lineare Räume. I”, Berlin, Springer, 1960.

[54] M. Lassak, Convex half-spaces, Fund. Math. 120 (1984), 7-13.

[55] M. Lassak, A. Prószynski, Translate-inclusive sets, orderings and convex half-spaces, Bull. Polish Acad. Sci. Math. 34 (1986), 195-201.

[56] M. LAssak, A. Prószynski, Algebraic and geometric approach to the classification of semispaces, Math. Scand. 61 (1987), 204-212.

[57] J. LaWrence, V. Soltan, On unions and intersections of nested families of cones, Beitr. Algebra Geom. 57 (2016), 655-665.

[58] K. Leichtweiss, "Konvexe Mengen", Springer, Berlin, 1980.

[59] J.E. Martínez-Legaz, Exact quasiconvex conjugation, Z. Oper. Res. Ser. $A-B 27$ (1983), A257-A266.

[60] J.E. Martínez-Legaz, I. Singer, Lexicographical separation in $R^{n}$, Linear Algebra Appl. 90 (1987), 147-163.

[61] J.E. Martínez-Legaz, I. Singer, The structure of hemispaces in $R^{n}$, Linear Algebra Appl. 110 (1988), 117-179.

[62] S. MazuR, Über konvexe Mengen in linearen normierten Räumen, Studia Math. 4 (1933), $70-84$.

[63] H. Minkowski, "Geometrie der Zahlen. I", Teubner, Leipzig, 1896; II. Teubner, Leipzig, 1910.

[64] H. Minkowski, "Gesammelte Abhandlungen. Bd 2", Teubner, Leipzig, 1911.

[65] C.E. Moore, Concrete semispaces and lexicographic order, Duke Math. J. 40 (1973), 53-61.

[66] Th. Motzkin, "Linear Inequalities. Mimeographed Lecture Notes", University of California, Los Angeles, CA, 1951.

[67] G. NöBeLING, Über die Konvexität von Raumstücken, Sitzungsber. Bayer. Akad. Wiss. Math.-Naturwiss. Kl. (1937), 63-67.

[68] Z. PÁLES, Separation theorems for convex sets and convex functions with invariance properties, Lecture Notes in Econom. and Math. Systems $\mathbf{5 0 2}$ (2001), 279-293.

[69] M.J. PAnIK, "Fundamentals of Convex Analysis", Kluwer, Dordrecht, 1993.

[70] K. Reinhardt, Über einen Satz von Herrn H. Tietze, Jahresber. Deutsch. Math.-Ver. 38 (1929), $191-192$. 
[71] K. REIDEMEISTER, Über die singulären Randpunkte eines konvexen Körpers, Math. Ann. 83 (1921), 116-118.

[72] R.T. Rockafellar, "Convex Analysis", Princeton University Press, Princeton, NJ, 1970.

[73] I. Singer, Generalized convexity, functional hulls and applications to conjugate duality in optimization, Lecture Notes in Econom. and Math. Systems 226 (1984), $49-79$.

[74] V. Soltan, Polarity and separation of cones, Linear Algebra Appl. 538 (2018), $212-224$

[75] V. Soltan, Asymptotic planes and closedness conditions, J. Convex Anal. 25 (2018), $1183-1196$

[76] V. Soltan, "Lectures on Convex Sets. Second Edition", World Scientific, Hackensack, NJ, 2020.

[77] V. Soltan, On M-decomposable sets, J. Math. Anal. Appl. 485 (2020), Paper No. $123816,15 \mathrm{pp}$.

[78] V. Soltan, Asymmetric separation of convex sets, Bul. Acad. Stiinţe Repub. Mold. Mat. 485 (2) (2020), 88-101.

[79] V. Soltan, On M-predecomposable sets, Beitr. Algebra Geom. 62 (2021), $205-218$.

[80] V. Soltan, Penumbras and separation of convex set, Results Math. 76 (2021), Paper No. 25, 21 pp.

[81] V. Soltan, Cone asymptotes of convex sets, Extracta Math. 36 (2021), 81-98.

[82] V. Soltan, Separating hyperplanes of convex sets, J. Convex Anal. 28 (2021), no. $4,20 \mathrm{pp}$.

[83] E. StEInitz, Bedingt konvergente Reihen und konvexe Systeme. I-IV, J. Reine Angew. Math. 143 (1913), 128-175.

[84] E. Steinitz, Bedingt konvergente Reihen und konvexe Systeme. VI-VII, J. Reine Angew. Math. 146 (1916), 1-52.

[85] M.H. Stone, "Convexity. Mimeographed Lectures. Fall Quarter 1946", University of Chicago, Chicago, IL, 1946.

[86] S. Straszewicz, "Beiträge zur Theorie der Konvexen Punktmengen", Inaugural Dissertation, Meier, Zürich, 1914.

[87] S. Straszewicz, Über exponierte Punkte abgeschlossener Punktmengen, Fund. Math. 24 (1935), 139-143.

[88] W. Süss, Eine Kennzeichnung von Eibereichen, Tôhoku Math. J. 32 (1930), $362-364$.

[89] H. Tietze, Über konvexe Figuren, J. Reine Angew. Math. 158 (1927), 168-172.

[90] H. Tietze, Eine charakteristische Eigenschaft der abgeschlossenen konvexen Punktmengen, Math. Ann. 99 (1928), 394-398.

[91] J.W. Tukey, Some notes on the separation of convex sets, Portugal. Math. 3 (1942), 95-102. 